PACS: $47.32 . \mathrm{C}$

\title{
NONLINEAR DYNAMO IN A ROTATING ELECTRICALLY CONDUCTING FLUID
}

\author{
M.I. Kopp ${ }^{1}$, A.V. Tur ${ }^{3}$, V.V. Yanovsky ${ }^{1,2}$ \\ ${ }^{1}$ Institute for Single Cristals, Nat. Academy of Science Ukraine, \\ Lenine Ave.60, Kharkov 31001, Ukraine \\ e-mail:yanovsky@isc.kharkov.ua \\ ${ }^{2}$ V.N. Karazin Kharkiv National University \\ Sq. Svobody 4, Kharkiv, 61022 Ukraine \\ ${ }^{3}$ Universite Toulouse [UPS], CNRS, Institute of Research for Astrophysics and Planetology \\ 9 avenue du Colonel Roche, BP 44346, 31028 Toulouse Cedex 4, France
}

Received January 21, 2017

We found a new large-scale instability, which arises in the rotating conductive fluid with small-scale turbulence. Turbulence is generated by small-scale external force with a low Reynolds number. The theory is built simply by the method of multiscale asymptotic expansions. Nonlinear equations for vortex and magnetic perturbations obtained in the third order for small Reynolds number. It is shown that the combined effects of the Coriolis force and the small external forces in a rotating conducting fluid possible large-scale instability. The large-scale increments of the instability, correspond to generation as the vortex and magnetic disturbances. This type of instability is classified as hydrodynamic and MHD alpha-effect. We studied the stationary regimes of nonlinear equations of magneto-vortex dynamo. In the limit of weakly conducting fluid found stationary solutions in the form of helical kinks. In the limit of high conductivity fluid was obtained stationary solutions in the form of nonlinear periodic waves and kinks.

KEY WORDS: equations of magnetohydrodynamics, Coriolis force, multiscale asymptotic expansions, small-scale turbulence, $\alpha-$ effect, spiral kinks

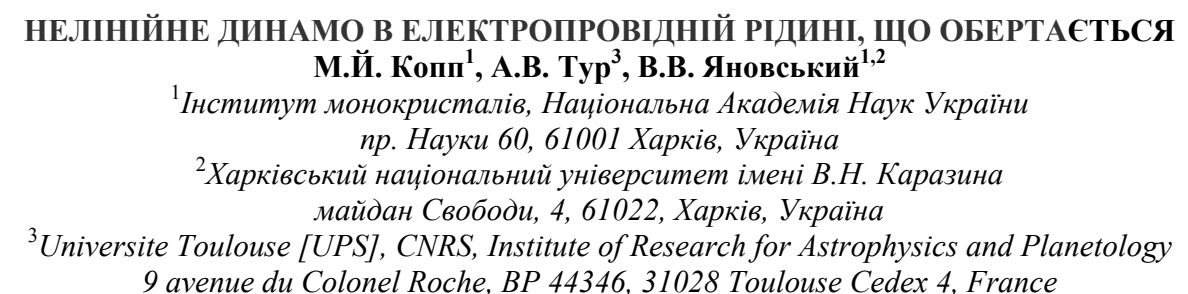

Знайдена нова великомасштабна нестійкість, виникаюча в електропровідній рідині, яка обертається 3 дрібномасштабною турбулентністю. Турбулентність генерується дрібномасштабною зовнішньою силою з малим числом Рейнольдса. Теорія побудована строгим методом великомасштабного асимптотичного розкладу. Нелінійні рівняння для вихрових і магнітних збурень отримані в третьому порядку по малому числу Рейнольдса. Показано, що в результаті спільної дії сили Коріоліса i дрібномасштабної зовнішньої сили в електропровідній рідині, яка обертається, можлива великомасшабна нестійкість. Отримані інкременти великомасштабної нестійкості, відповідні генерації як вихрових, так і магнітних збурень. Такий тип нестійкості класифікується як гідродинамічний і магнітогідродинамічний альфа-ефект. Було вивчено стаціонарні режими нелінійних рівнянь магніто-вихрового динамо. В межі слабкопровідної рідини знайдені стаціонарні рішення у вигляді спіральних кінків. В межі високої провідності рідини отримані стаціонарні рішення у вигляді нелінійних періодичних хвиль і кінків.

КЛЮЧОВІ СЛОВА: рівняння магнітної гідродинаміки, сила Коріоліса, великомасштабні асимптотичні розкладання, дрібномасштабна турбулентність, $\alpha$-ефект, спіральні кінки

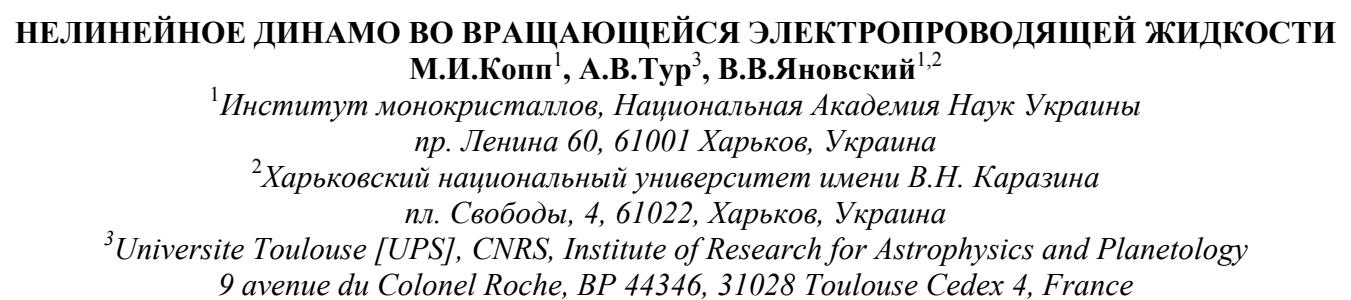

Найдена новая крупномасштабная неустойчивость, которая возникает во вращающейся электропроводящей жидкости с мелкомасштабной турбулентностью. Турбулентность генерируется мелкомасштабной внешней силой с малым числом Рейнольдса. Теория построена строгим методом многомасштабного асимптотического разложения. Нелинейные уравнения для вихревых и магнитных возмущений получены в третьем порядке по малому числу Рейнольдса. Показано, что в результате совместного действия силы Кориолиса и мелкомасштабной внешней силы во вращающейся электропроводящей жидкости возможна крупномасштабная неустойчивость. Получены инкременты крупномасштабной неустойчивости, соответствующие генерации как вихревых, так и магнитных возмущений. Такой тип неустойчивости классифицируется как гидродинамический и магнитогидродинамический альфа-эффект. Изучены стационарные режимы нелинейных уравнений магнито-вихревого динамо. В пределе слабопроводящей жидкости найдены стационарные решения в виде спиральных 
кинков. В пределе высокой проводимости жидкости получены стационарные решения в виде нелинейных периодических волн и кинков.

КЛЮЧЕВЫЕ СЛОВА: уравнения магнитной гидродинамики, сила Кориолиса, многомасштабные асимптотические разложения, мелкомасштабная турбулентность, $\alpha$-эффект, спиральные кинки

Как известно, исследованием проблемы происхождения магнитных полей у астрофизических объектов таких как планеты, Солнце, галактики занимается теория динамо [1-10]. Огромную роль здесь играет вращательное движение космических тел, благодаря которому возбуждаются различные волновые и вихревые движения такие как гироскопические волны, волны Россби, внутренние волны, локализованные вихри и когерентные структуры [11-18]. Эффект вращения также оказывает существенное влияние на турбулентное движение жидкости (см. например [19]). Природа такого влияния связана с действием силы Кориолиса, которая нарушает зеркальную симметрию турбулентного движения жидкости. Турбулентность с таким свойством получила название спиральной. Именно в спиральной турбулентности сначала был открыт $\alpha$-эффект в электропроводящей жидкости или магнитной гидродинамике (МГД) [20], а затем в обычной гидродинамике (ГД) [20]. В МГД спиральная турбулентность генерирует крупномасштабное магнитное поле, а в ГД крупномасштабные вихревые структуры. Изначально теория $\alpha$-эффекта в МГД строилась в кинематической постановке, т.е. когда не учитывается обратное воздействие магнитного поля на турбулентное течение. В этом случае генерация среднего (крупномасштабного) магнитного поля происходит под действием средней э.д.с которая пропорциональна среднему магнитному полю $\overrightarrow{\vec{H}}: \overrightarrow{\mathcal{E}}=\alpha \overrightarrow{\vec{H}}$. Коэффициент усиления $\alpha$ пропорционален средней спиральности поля скорости $\alpha \sim \overrightarrow{\vec{v}} \operatorname{rot} \vec{v}$, которая характеризует меру заузленности вихревых движений среды. Как показано в работе [22] ненулевая средняя спиральность не является необходимым условием для появления $\alpha$ - эффекта, но способствует его возникновению. Несмотря на формальную аналогию уравнения индукции для магнитного поля $\vec{H}$ и уравнения для завихренности $\vec{\omega}=\operatorname{rot} \vec{v}$, перенос $\alpha$-эффекта на Гд сталкивается с дополнительной проблемой. Причина отрицательного эффекта заключается в определенной симметрии тензора напряжений Рейнольдса в осредненном уравнении Навье-Стокса [23]. Оказалось, что для появления ГД $\alpha$ - эффекта одной спиральности турбулентности не достаточно, нужны другие факторы нарушения симметрии турбулентного течения. Такими факторами являются сжимаемость среды [21], неоднородный поток [24], градиент температуры в поле тяжести [25]. Эффект генерации крупномасштабных вихревых структур (КВС) спиральной турбулентностью получил название вихревого динамо. Механизмы вихревого динамо развивались для описания разнообразных явлений в турбулентной атмосфере и океане. Особое внимание уделялось конвективному вихревому динамо [25-32], где спиральная турбулентность приводила к крупномасштабной неустойчивости, вследствие которой образуется одна конвективная ячейка, интерпретируемая как огромный вихрь типа тропического циклона. Известно также большое количество работ по генерации КВС с учетом эффектов вращения [33-37]. Принципиально отличный $\alpha$ - эффект был обнаружен в работе [38], в которой турбулентное движение жидкости моделируется внешней мелкомасштабной силой $\vec{F}_{0}$. Модель внешней мелкомасштабной силы была выбрана с нарушением четности (при нулевой спиральности $\vec{F}_{0} r o t \vec{F}_{0}=0$ ). Эффект генерации крупномасштабных возмущений такой силой получил название анизотропного кинетического альфа-эффекта или АКА-эффекта [38]. Отметим, что нарушение четности более общее явление, чем спиральность, хотя именно спиральность $\overrightarrow{\vec{v} r o t \vec{v}} \neq 0$ является самым распространенным механизмом нарушения четности гидродинамических течений. В этой же работе была рассмотрена крупномасштабная неустойчивость в несжимаемой жидкости методом асимптотических многомасштабных разложений. В качестве малого параметра для асимптотического метода многомасштабных разложений используется число Рейнольдса $R=\frac{v_{0} t_{0}}{\lambda_{0}} \ll 1$ для мелкомасштабных пульсаций скорости $v_{0}$, вызванных мелкомасштабной силой. В дальнейшем, применяя метод многомасштабных асимптотических разложений были разработаны линейные и нелинейные теории вихревого динамо для сжимаемых сред [39, 40], конвективных сред со спиральной внешней силой [30-32]. Новый пример генерации КВС во вращающейся несжимаемой жидкости был найден в работе [41]. Там же было показано, что в результате развития крупномасштабной неустойчивости во вращающейся жидкости возникают нелинейные крупномасштабные спиральные вихревые структуры типа вихрей Бельтрами или локализованные кинки с внутренней спиральной структурой. Обобщение этого ГД $\alpha$ - эффекта на случай электропроводящей жидкости приводит к новым крупномасштабным структурам.

В этой работе получена крупномасштабная неустойчивость, приводящая к генерации КВС и магнитных полей и формированию крупномасштабных нелинейных стационарных структур. В разделе ОСНОВНЫЕ УРАВНЕНИЯ И ПОСТАНОВКА ЗАДАЧИ приведены основные уравнения и сформулирована постановка задачи, исследуемой в настоящей работе. В разделе УРАВНЕНИЯ НЕЛИНЕЙНОГО МАГНИТО- 
ВИХРЕВОГО ДИНАМО В «КВАЗИДВУМЕРНОЙ» МОДЕЛИ, ПрИменяя метод многомасштабнЫХ асимптотических разложений, получены осредненные уравнения МГД во вращающейся жидкости для крупномасштабных полей. Алгебраическая структура уравнений многомасштабного асимптотического разложения в различных порядках по $R$ приведена в Приложении I. Корреляционные функции, входящие в осредненные уравнения, выражаются через мелкомасштабные поля в нулевом приближении по $R$. В Приложении II получены решения уравнений для мелкомасштабных полей в нулевом порядке по $R$. Используя эти решения, в Приложении III вычислены корреляционные функции, соответствующие напряжениям Рейнольдса, напряжениям Максвелла и турбулентной э.д.с. В результате, в разделе УРАВНЕНИЯ НЕЛИНЕЙНОГО МАГНИТО-ВИХРЕВОГО ДИНАМО В «КВАЗИДВУМЕРНОЙ» МОДЕЛИ получена самосогласованная система нелинейных уравнений для крупномасштабных магнитных и гидродинамических полей. В отличие от кинематического динамо, эти поля оказывают взаимное влияние друг на друга. Система уравнений, описывающая взаимное влияние полей, названа уравнениями нелинейного магнито-вихревого динамо. В разделе КРУПНОМАСШТАБНАЯ НЕУСТОЙЧИВОСТЬ исследуется устойчивость малых крупномасштабных вихревых и магнитных возмущений. В разделе НЕЛИНЕЙНЫЕ СТАЦИОНАРНЫЕ СТРУКТУРЫ проведен численный анализ нелинейных уравнений в стационарном режиме. Там же показано существование локализованных вихревых и магнитных структур.

Целью настоящей работы является исследование линейной и нелинейной генерации крупномасштабных вихревых и магнитных полей во вращающихся электропроводных турбулентных средах. Полученные в работе результаты могут найти применение во многих астрофизических задачах.

\section{ОСНОВНЫЕ УРАВНЕНИЯ И ПОСТАНОВКА ЗАДАЧИ}

Исходными уравнениями для описания динамики вращающейся электропроводящей несжимаемой жидкости являются хорошо известные уравнения одножидкостной магнитной гидродинамики:

$$
\begin{gathered}
\frac{\partial \vec{V}}{\partial t}+(\vec{V} \nabla) \vec{V}=-\frac{\nabla P}{\rho_{00}}+2[\vec{V} \times \vec{\Omega}]+\frac{1}{4 \pi \rho_{00}}[\operatorname{rot} \vec{B} \times \vec{B}]+v \Delta \vec{V}+\vec{F}_{0}, \\
\frac{\partial \vec{B}}{\partial t}=\operatorname{rot}[\vec{V} \times \vec{B}]+v_{m} \Delta B, \\
\operatorname{div} \vec{V}=0, \operatorname{div} \vec{B}=0 .
\end{gathered}
$$

Здесь $\vec{V}, P, \vec{B}$ - возмущения скорости, давления и индукции магнитного поля относительно равновесного состояния:

$$
\nabla P_{00}=-\rho_{00} \nabla \Phi_{00}-\rho_{00}[\vec{\Omega} \times[\vec{\Omega} \times \vec{r}]],
$$

где $\vec{r}$ - радиус-вектор элемента среды, $\Phi_{00}$ - равновесный потенциал, описывающий внешнюю силу тяжести, $v$ - коэффициент кинематической вязкости жидкости, $v_{m}=\frac{c^{2}}{4 \pi \sigma_{c}}$ - коэффициент магнитной вязкости, $\sigma_{c}$ коэффициент электропроводности среды. Вектор угловой скорости вращения $\vec{\Omega}$ для простоты считаем постоянным (твердотельное вращение) и в декартовой системе координат: $\vec{\Omega}=\left(\Omega_{1}, \Omega_{2}, \Omega_{3}\right)$. Кроме того, среду будем считать безграничной и влиянием внешнего магнитного поля пренебрежем. В этом случае мелкомасштабные магнитные поля или так называемые «затравочные» магнитные поля могут возбуждаться не турбулентными механизмами, а например в результате развития гидродинамических неустойчивостей [1-3], термомагнитных неустойчивостей [42]. Такая постановка задачи интересна для теории динамо [1-10]. В уравнение (1) включена внешняя сила $\vec{F}_{0}$, моделирующая источник возбуждения в среде мелкомасштабных и высокочастотных флуктуаций поля скорости $\vec{v}_{0}$ с малым числом Рейнольдса $R=\frac{v_{0} t_{0}}{\lambda_{0}} \ll 1$ :

$$
\operatorname{div} \vec{F}_{0}=0, \vec{F}_{0}=f_{0} \vec{F}_{0}\left(\frac{x}{\lambda_{0}}, \frac{t}{t_{0}}\right),
$$

где $\lambda_{0}$ - характерный масштаб, $t_{0}$ - характерное время, $f_{0}$ - характерная амплитуда. Перейдем в уравнениях (1)(3) к безразмерным переменным. Чтобы не загромождать обозначения сохраним за безразмерными переменными обозначения прежних размерных переменных. Это не вызывает в дальнейшем никаких затруднений. 


$$
\begin{aligned}
& t \rightarrow \frac{t}{t_{0}}, \quad \vec{V} \rightarrow \frac{\vec{V}}{v_{0}}, \quad \vec{F}_{0} \rightarrow \frac{\vec{F}_{0}}{f_{0}}, \quad \vec{B} \rightarrow \frac{\vec{B}}{B_{0}}, \\
& \vec{x} \rightarrow \frac{\vec{x}}{\lambda_{0}}, t_{0}=\frac{\lambda_{0}^{2}}{v}, \quad f_{0}=\frac{v_{0} v}{\lambda_{0}^{2}}, \quad P \rightarrow \frac{P}{P_{0} \rho_{00}}, \quad P_{0}=\frac{v v_{0}}{\lambda_{0}} .
\end{aligned}
$$

В безразмерных переменных уравнения (1)-(3) примут вид:

$$
\begin{gathered}
\frac{\partial V_{i}}{\partial t}+R V_{k} \frac{\partial V_{i}}{\partial x_{k}}=-\frac{1}{\rho_{00}} \frac{\partial P}{\partial x_{i}}+\varepsilon_{i j k} V_{j} D_{k}+\widetilde{Q} R \varepsilon_{i j k} \varepsilon_{j m l} \frac{\partial B_{l}}{\partial x_{m}} B_{k}+\frac{\partial^{2} V_{i}}{\partial x_{k}^{2}}+F_{0}^{i}, \\
\frac{\partial B_{i}}{\partial t}-P m^{-1} \frac{\partial^{2} B_{i}}{\partial x_{k}^{2}}=R \varepsilon_{i j k} \varepsilon_{k n p} V_{n} B_{p}, \\
\frac{\partial V_{k}}{\partial x_{k}}=\frac{\partial B_{k}}{\partial x_{k}}=0 .
\end{gathered}
$$

Характер эволюции полей, описываемых системой уравнений (7)-(9), в значительной степени будет определяться следующими безразмерными параметрами: $D_{i}=\frac{2 \Omega_{i} \lambda_{0}^{2}}{v}-$ безразмерный параметр вращения $(i=1,2,3)$ на масштабе $\lambda_{0}$, связанный с числом Тейлора $T a_{i}=D_{i}^{2}$ [11-13]. Этот безразмерный параметр характеризует степень превалирования сил Кориолиса над вязкими силами. $\widetilde{Q}=\frac{Q}{P m}, Q=\frac{\sigma_{c} B_{0}^{2} \lambda_{0}^{2}}{c^{2} \rho_{00} v}$ - число Чандрасекара, $P m=\frac{v}{v_{m}}$ - магнитное число Прандтля. Малым параметром асимптотического разложения считаем число Рейнольдса $R=\frac{v_{0} t_{0}}{\lambda_{0}} \ll 1$ мелкомасштабных движений, а параметры $D$ и $\widetilde{Q}$ произвольными, не влияющими на схему разложения. Рассмотрим следующую постановку задачи. Пусть внешняя сила на фоне равновесного состояния вызывает мелкомасштабные и высокочастотные осцилляции скорости. Средние значения таких осцилляций нулевые, но из-за нелинейного взаимодействия в некоторых порядках теории возмущения возникают члены, которые при усреднении не обращаются в нуль. Такие члены называются секулярными и являются условиями разрешимости многомасштабного асимптотического разложения. Нахождение уравнений разрешимости, которые и определяют эволюцию крупномасштабных возмущений, является основной задачей.

\section{УРАВНЕНИЯ НЕЛИНЕЙНОГО МАГНИТО-ВИХРЕВОГО ДИНАМО В «КВАЗИДВУМЕРНОЙ»} МОДЕЛИ

Рассмотрим более детально применение метода многомасштабных асимптотических разложений к проблеме нелинейной эволюции крупномасштабных вихревых и магнитных возмущений во вращающейся электропроводящей среде. Метод построения асимптотических уравнений развит в работах [30-32,38], следуя которым представим пространственные и временные производные в уравнениях (7)-(9) в виде:

$$
\frac{\partial}{\partial t} \rightarrow \partial_{t}+R^{4} \partial_{T}, \frac{\partial}{\partial x_{i}} \rightarrow \partial_{i}+R^{2} \nabla_{i}
$$

где $\partial_{i}$ и $\partial_{t}$ - обозначают производные по быстрым переменным $x_{0}=\left(\vec{x}_{0}, t_{0}\right)$, а $\nabla_{i}$ и $\partial_{T}$ - производные по медленным переменным $X=(\vec{X}, T)$. Переменные $x_{0}$ и $X$ соответственно можно назвать мелкомасштабные и крупномасштабные переменные. При построении нелинейной теории переменные $\vec{V}, \vec{B}, P$ представим в виде асимптотического ряда:

$$
\begin{aligned}
& \vec{V}(\vec{x}, t)=\frac{1}{R} \vec{W}_{-1}(X)+\vec{v}_{0}\left(x_{0}\right)+R \vec{v}_{1}+R^{2} \vec{v}_{2}+R^{3} \vec{v}_{3}+\cdots \\
& \vec{B}(\vec{x}, t)=\frac{1}{R} \vec{B}_{-1}(X)+\vec{B}_{0}\left(x_{0}\right)+R \vec{B}_{1}+R^{2} \vec{B}_{2}+R^{3} \vec{B}_{3}+\cdots \\
& P(x)=\frac{1}{R^{3}} P_{-3}+\frac{1}{R^{2}} P_{-2}+\frac{1}{R} P_{-1}+P_{0}+R\left(P_{1}+\bar{P}_{1}(X)\right)+R^{2} P_{2}+R^{3} P_{3}+\cdots
\end{aligned}
$$

Подставим разложения (10)-(11) в систему уравнений (7)-(9) и зануляя вклады в каждом порядке по $R$ до 
степени $R^{3}$ включительно, получим уравнения многомасштабного асимптотического разложения. Алгебраическая структура асимптотического разложения уравнений (7)-(9) в различных порядках по $R$ приведена в Приложении I. Там же показано, что именно в порядке $R^{3}$ получаются основные секулярные уравнения, т.е. уравнения для крупномасштабных полей:

$$
\begin{gathered}
\partial_{t} W_{-1}^{i}-\Delta W_{-1}^{i}+\nabla_{k} \overline{\left(v_{0}^{k} v_{0}^{i}\right)}=-\nabla_{i} \bar{P}_{1}+\widetilde{Q} \varepsilon_{i j k} \varepsilon_{j m l}\left(\nabla_{m} \overline{\left(B_{0}^{l} B_{0}^{k}\right)}\right), \\
\partial_{t} B_{-1}^{i}-P m^{-1} \Delta B_{-1}^{i}=\varepsilon_{i j k} \varepsilon_{k n p} \nabla_{j} \overline{\left(v_{0}^{n} B_{0}^{p}\right)} .
\end{gathered}
$$

Используя свертку тензоров $\varepsilon_{i j k} \varepsilon_{j m l}=\delta_{k m} \delta_{i l}-\delta_{i m} \delta_{k l}, \varepsilon_{i j k} \varepsilon_{k n p}=\delta_{i n} \delta_{j p}-\delta_{i p} \delta_{j p} \quad$ и вводя обозначения $\vec{W}=\vec{W}_{-1}, \vec{H}=\vec{B}_{-1}$ система уравнений (12)-(13) примет следующий вид:

$$
\begin{gathered}
\partial_{T} W_{i}-\Delta W_{i}+\nabla_{k} \overline{\left(v_{0}^{k} v_{0}^{i}\right)}=-\nabla_{i} \bar{P}_{1}+\widetilde{Q}\left(\nabla_{k} \overline{\left(B_{0}^{i} B_{0}^{k}\right)}-\frac{\nabla_{i}}{2} \overline{\left(B_{0}^{k}\right)^{2}}\right), \\
\partial_{T} H_{i}-P m^{-1} \Delta H_{i}=\nabla_{j} \overline{\left(v_{0}^{i} B_{0}^{j}\right)}-\nabla_{j} \overline{\left(v_{0}^{j} B_{0}^{i}\right)} .
\end{gathered}
$$

Уравнения (14)-(15) дополняются секулярными уравнениями, которые были получены в Приложении I:

$$
\begin{gathered}
\nabla_{k}\left(W_{k} W_{i}\right)=-\nabla_{i} P_{-1}+\widetilde{Q}\left(\nabla_{k} H_{i}-\nabla_{i} H_{k}\right) H_{k}, \\
W_{j} \nabla_{j} H_{i}=H_{j} \nabla_{j} W_{i}, \\
W_{j} \nabla_{j} H_{i}=H_{j} \nabla_{j} W_{i}, \\
\nabla_{i} W_{i}=0, \nabla_{i} H_{i}=0, \nabla_{i} P_{-3}=\varepsilon_{i j k} W_{j} D_{k} .
\end{gathered}
$$

Таким образом для получения системы уравнений (14)-(15), описывающей эволюцию крупномасштабных полей $\vec{W}$ и $\vec{H}$ потребовалось дойти до третьего порядка теории возмущения. Это довольно характерное явление при применении метода многомасштабных разложений. Уравнения (14)-(15) приобретают замкнутый вид после вычисления корреляционных функций -- напряжений Рейнольдса $\nabla_{k} \overline{\left(v_{0}^{k} v_{0}^{i}\right)}$, напряжений Максвелла $\nabla_{k} \overline{\left(B_{0}^{i} B_{0}^{k}\right)}$ и турбулентной э.д.с. $\mathcal{E}_{n}=\varepsilon_{n i j} \overline{v_{0}^{i} B_{0}^{j}}$. Вычисление этих корреляционных функций значительно упрощается, если воспользоваться «квазидвумерным» приближением, которое часто применяется для описания крупномасштабных вихревых и магнитных полей во многих астрофизических и геофизических задачах $[3,14,30,31]$. В рамках этого приближения для нашей задачи будем считать, что крупномасштабная производная по $Z$ более предпочтительная, т.е.

$$
\frac{\partial}{\partial Z} \gg \frac{\partial}{\partial X}, \frac{\partial}{\partial Y},
$$

а геометрия крупномасштабных полей имеет следующий вид:

$$
\vec{W}=\left(W_{1}(Z), W_{2}(Z), 0\right), \vec{H}=\left(H_{1}(Z), H_{2}(Z), 0\right) .
$$

В рамках «квазидвумерной» задачи система уравнений (14)-(15) упрощается:

$$
\begin{aligned}
& \partial_{T} W_{1}-\Delta W_{1}+\nabla_{Z} \overline{\left(v_{0}^{z} v_{0}^{x}\right)}=\widetilde{Q} \nabla_{Z} \overline{\left(B_{0}^{z} B_{0}^{x}\right)}, \\
& \partial_{T} W_{2}-\Delta W_{2}+\nabla_{Z} \overline{\left(v_{0}^{z} v_{0}^{y}\right)}=\widetilde{Q} \nabla_{Z} \overline{\left(B_{0}^{z} B_{0}^{y}\right)}, \\
& \partial_{T} H_{1}-P m^{-1} \Delta H_{1}=\nabla_{Z} \overline{\left(v_{0}^{x} B_{0}^{z}\right)}-\nabla_{Z} \overline{\left(v_{0}^{z} B_{0}^{x}\right)}, \\
& \partial_{T} H_{2}-P m^{-1} \Delta H_{2}=\nabla_{Z} \overline{\left(v_{0}^{y} B_{0}^{z}\right)}-\nabla_{Z} \overline{\left(v_{0}^{z} B_{0}^{y}\right)} .
\end{aligned}
$$

Для получения уравнений (17)-(20) в замкнутом виде мы используем решения уравнений для мелкомасштабных полей в нулевом порядке по $R$, полученные в Приложении II. Далее необходимо вычислить корреляторы, входящие в систему уравнений (17)-(20):

$$
\begin{gathered}
T^{31}=\overline{w_{0} u_{0}}=\overline{w_{01}\left(u_{01}\right)^{*}}+\overline{\left(w_{01}\right)^{*} u_{01}}+\overline{w_{03}\left(u_{03}\right)^{*}}+\overline{\left(w_{03}\right)^{*} u_{03}}, \\
T^{32}=\overline{w_{0} v_{0}}=\overline{w_{01}\left(v_{01}\right)^{*}}+\overline{\left(w_{01}\right)^{*} v_{01}}+\overline{w_{03}\left(v_{03}\right)^{*}}+\overline{\left(w_{03}\right)^{*} v_{03},} \\
S^{31}=\overline{\widetilde{w}_{0} \tilde{u}_{0}}=\overline{\widetilde{w}_{01}\left(\tilde{u}_{01}\right)^{*}}+\overline{\left(\tilde{w}_{01}\right)^{*} \tilde{u}_{01}}+\overline{\widetilde{w}_{03}\left(\tilde{u}_{03}\right)^{*}}+\overline{\left(\tilde{w}_{03}\right)^{*} \tilde{u}_{03},}
\end{gathered}
$$




$$
\begin{aligned}
& S^{32}=\overline{\widetilde{w}_{0} \tilde{v}_{0}}=\overline{\widetilde{w}_{01}\left(\tilde{v}_{01}\right)^{*}}+\overline{\left(\widetilde{\left.w_{01}\right)^{*} \tilde{v}_{01}}\right.}+\overline{\widetilde{w}_{03}\left(\tilde{v}_{03}\right)^{*}}+\overline{\left(\tilde{w}_{03}\right)^{*} \tilde{v}_{03}},
\end{aligned}
$$

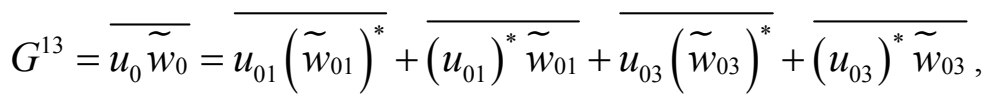

$$
\begin{aligned}
& G^{31}=\overline{w_{0} \tilde{u}_{0}}=\overline{w_{01}\left(\tilde{u}_{01}\right)^{*}}+\overline{\left(w_{01}\right)^{*} \tilde{u}_{01}}+\overline{w_{03}\left(\tilde{u}_{03}\right)^{*}}+\overline{\left(w_{03}\right)^{*} \tilde{u}_{03}},
\end{aligned}
$$

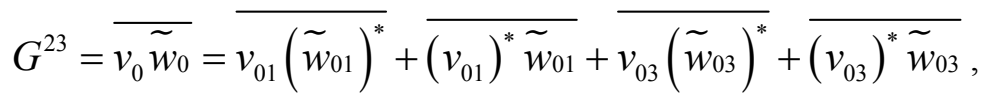

$$
\begin{aligned}
& G^{32}=\overline{w_{0} \tilde{v}_{0}}=\overline{w_{01}\left(\tilde{v}_{01}\right)^{*}}+\overline{\left(w_{01}\right)^{*} \tilde{v}_{01}}+\overline{w_{03}\left(\tilde{v}_{03}\right)^{*}}+\overline{\left(w_{03}\right)^{*} \tilde{v}_{03}} .
\end{aligned}
$$

Здесь для удобства приняты новые обозначения мелкомасштабных полей: $v_{0}^{x}=u_{0}, v_{0}^{y}=v_{0}, v_{0}^{z}=w_{0}$, $B_{0}^{x}=\widetilde{u}_{0}, B_{0}^{y}=\widetilde{v}_{0}, B_{0}^{z}=\widetilde{w}_{0}$. Техническая сторона этого вопроса подробно изложена в Приложении III. В результате проведенных там вычислений, мы получили замкнутые уравнения для крупномасштабных полей скорости $\left(W_{1}, W_{2}\right)$ и магнитного поля $\left(H_{1}, H_{2}\right)$ следующего вида:

$$
\begin{aligned}
& \partial_{T} W_{1}-\Delta W_{1}+\nabla_{Z}\left(\alpha_{(1)} \cdot\left(1-W_{2}\right)\right)=0, \\
& \partial_{T} W_{2}-\Delta W_{2}-\nabla_{Z}\left(\alpha_{(2)} \cdot\left(1-W_{1}\right)\right)=0, \\
& \partial_{T} H_{1}-P m^{-1} \Delta H_{1}+\nabla_{Z}\left(\alpha_{H}^{(2)} H_{2}\right)=0, \\
& \partial_{T} H_{2}-P m^{-1} \Delta H_{2}-\nabla_{Z}\left(\alpha_{H}^{(1)} H_{1}\right)=0 .
\end{aligned}
$$

Уравнения (21)-(24) описывают нелинейную динамику крупномасштабных полей во вращающейся электропроводящей среде. Связь между компонентами крупномасштабного вихревого и магнитного поля осуществляется при помощи коэффициентов нелинейного гидродинамического (ГД) $\alpha_{(1)}, \alpha_{(2)}$ и магнитогидродинамического (МГД) $\alpha_{H}^{(1)}, \alpha_{H}^{(2)} \alpha$-эффекта. Причем коэффициенты нелинейного ГД и МГД $\alpha$-эффекта являются функциями крупномасштабных полей скорости $\vec{W}$ и магнитного поля $\vec{H}$ :

$$
\begin{aligned}
& \alpha_{(1)}=\frac{f_{0}^{2}}{2} \frac{D q_{2}\left(1+P m\left(1-q_{2}\right)\right)\left(1-W_{2}\right)^{-1}}{\left[16\left(1-W_{2}\right)^{2}+\left[\frac{D^{2}}{2}+4-\left(1-W_{2}\right)^{2}\right]^{2}+h_{2}\right]}- \\
& -\frac{f_{0}^{2}}{8} \frac{D^{2}\left(1+P m\left(1-q_{1}\right)\right)\left(1-W_{2}\right)^{-1}}{\left[16\left(1-W_{1}\right)^{2}+\left[\frac{D^{2}}{2}+4-\left(1-W_{1}\right)^{2}\right]^{2}+h_{1}\right]} \\
& \alpha_{(2)}=\frac{f_{0}^{2}}{2} \frac{D q_{1}\left(1+\operatorname{Pm}\left(1-q_{1}\right)\right)\left(1-W_{1}\right)^{-1}}{\left[16\left(1-W_{1}\right)^{2}+\left[\frac{D^{2}}{2}+4-\left(1-W_{1}\right)^{2}\right]^{2}+h_{1}\right]}+ \\
& +\frac{f_{0}^{2}}{8} \frac{D^{2}\left(1+P m\left(1-q_{2}\right)\right)\left(1-W_{1}\right)^{-1}}{\left[16\left(1-W_{2}\right)^{2}+\left[\frac{D^{2}}{2}+4-\left(1-W_{2}\right)^{2}\right]^{2}+h_{2}\right]}, \\
& \alpha_{H}^{(1)}=f_{0}^{2} \frac{D\left(1-W_{1}\right) \operatorname{Pm}\left(1+\operatorname{Pm}\left(1-q_{1}\right)\right) H_{1}}{\left(4+P m^{2}\left(1-W_{1}\right)^{2}\right)\left[16\left(1-W_{1}\right)^{2}+\left[\frac{D^{2}}{2}+4-\left(1-W_{1}\right)^{2}\right]^{2}+h_{1}\right]} \text {, } \\
& \alpha_{H}^{(2)}=f_{0}^{2} \frac{D\left(1-W_{2}\right) P m\left(1+P m\left(1-q_{2}\right)\right) H_{2}}{\left(4+P m^{2}\left(1-W_{2}\right)^{2}\right)\left[16\left(1-W_{2}\right)^{2}+\left[\frac{D^{2}}{2}+4-\left(1-W_{2}\right)^{2}\right]^{2}+h_{2}\right]} \text {. }
\end{aligned}
$$


Здесь приняты следующие обозначения:

$$
\begin{aligned}
& q_{1,2}=1+\frac{Q H_{1,2}^{2}}{4+P m^{2}\left(1-W_{1,2}\right)^{2}}, \\
& h_{1,2}=2 f_{1,2}\left(4+\left(1-W_{1,2}\right)^{2}\right)^{2}+f_{1,2}^{2}\left(4+\left(1-W_{1,2}\right)^{2}\right)^{2}+D^{2} g_{1,2}, \\
& g_{1,2}=\frac{Q H_{1,2}^{2}}{\left(4+P m^{2}\left(1-W_{1,2}\right)^{2}\right)}\left[2\left(4+P m\left(1-W_{1,2}\right)^{2}\right)+\frac{Q H_{1,2}^{2}\left(4-P m^{2}\left(1-W_{1,2}\right)^{2}\right)}{4+P m^{2}\left(1-W_{1,2}\right)^{2}}\right], \\
& f_{1,2}=\frac{Q H_{1,2}^{2}}{\left(4+\left(1-W_{1,2}\right)^{2}\right)\left(4+P m^{2}\left(1-W_{1,2}\right)^{2}\right)}\left[2\left(4+P m\left(1-W_{1,2}\right)^{2}\right)+Q H_{1,2}^{2}\right] .
\end{aligned}
$$

Таким образом в данном разделе мы получили замкнутые уравнения (29)-(32) нелинейного магнитовихревого динамо во вращающейся электропроводящей жидкости с внешней мелкомасштабной силой $\vec{F}_{0}$. Если эффект вращения исчезнет ( $\Omega=0$ ), то произойдет обычное диффузионное расплывание крупномасштабных полей. В пределе неэлектропроводящей жидкости $\sigma=0$ уравнения (29), (30) полностью совпадают с результатами работы [41]. Далее рассмотрим устойчивость малых возмущений полей (линейная теория), а затем рассмотрим условия существования стационарных нелинейных структур.

\section{КРУПНОМАСШТАБНАЯ НЕУСТОЙЧИВОСТЬ}

Рассмотрим начальную стадию развития возмущений $\left(W_{1}, W_{2}\right)$ и $\left(H_{1}, H_{2}\right)$. Тогда при малых значениях величин $\left(W_{1}, W_{2}\right)$ и $\left(H_{1}, H_{2}\right)$ уравнения (29)-(32) линеаризуются и сводятся к следующей системе линейных уравнений:

$$
\begin{aligned}
& \left\{\begin{array}{l}
\partial_{T} W_{1}-\nabla_{Z}^{2} W_{1}-\alpha_{1} \nabla_{Z} W_{1}+\alpha_{2} \nabla_{Z} W_{2}=0 \\
\partial_{T} W_{2}-\nabla_{Z}^{2} W_{2}-\alpha_{1} \nabla_{Z} W_{2}-\alpha_{2} \nabla_{Z} W_{1}=0
\end{array},\right. \\
& \left\{\begin{array}{l}
\partial_{T} H_{1}-P m^{-1} \nabla_{Z}^{2} H_{1}+\alpha_{H} \nabla_{Z} H_{2}=0 \\
\partial_{T} H_{2}-P m^{-1} \nabla_{Z}^{2} H_{2}-\alpha_{H} \nabla_{Z} H_{1}=0
\end{array}\right.
\end{aligned}
$$

где введены следующие обозначения для коэффициентов:

$$
\begin{array}{r}
\alpha_{1}=\frac{f_{0}^{2}}{8} \frac{32 D^{2}\left(10-D^{2}\right)}{\left(\left(D^{2}+6\right)^{2}+64\right)^{2}}, \\
\alpha_{2}=\frac{f_{0}^{2}}{2} \frac{32 D\left(10-D^{2}\right)}{\left(\left(D^{2}+6\right)^{2}+64\right)^{2}}, \\
\alpha_{H}=f_{0}^{2} \frac{4 P m D}{\left(4+P m^{2}\right)\left(\left(D^{2}+6\right)^{2}+64\right)} .
\end{array}
$$

Как видно из системы уравнений (25)-(26) при малых возмущениях полей происходит расщепление самосогласованной системы уравнений (21)-(24) на две пары уравнений для крупномасштабного поля скорости $\vec{W}$ и магнитного поля $\vec{H}$ соответственно. Первая пара уравнений (25) подобна уравнениям для вихревого динамо [21,25], отличие от которых состоит в появлении дополнительного конвективного члена с $\alpha_{1}$. Посредством коэффициента $\alpha_{2}$ устанавливается положительная обратная связь с помощью которой возможна генерация крупномасштабных вихревых структур. Вторая пара уравнений (26) описывает хорошо известный $\alpha$-эффект в теории динамо [1-10] или усиление крупномасштабного магнитного поля мелкомасштабной спиральной турбулентностью. Для исследования крупномасштабной неустойчивости, описываемой системой уравнений (25)-(26), выберем возмущения в виде плоских волн с волновым вектором $\vec{K} \| O Z$, т.е. 


$$
\left(\begin{array}{c}
W_{1,2} \\
H_{1,2}
\end{array}\right)=\left(\begin{array}{c}
A_{W_{1,2}} \\
A_{H_{1,2}}
\end{array}\right) \exp (-i \omega T+i K Z) .
$$

Подставим (30) в систему уравнений (25), (26) получим дисперсионные уравнения:

$$
\left(-i \omega+K^{2}-i \alpha_{1} K\right)^{2}-\alpha_{2}^{2} K^{2}=0,\left(-i \omega+P m^{-1} K^{2}\right)^{2}-\alpha_{H}^{2} K^{2}=0 .
$$

Представив $\omega=\omega_{0}+i \Gamma$ из уравнений (31) находим:

$$
\begin{aligned}
& \omega_{01}=\operatorname{Re} \omega_{1}=-\alpha_{1} K, \Gamma_{1}=\operatorname{Im} \omega_{1}= \pm \alpha_{2} K-K^{2} \\
& \omega_{02}=\operatorname{Re} \omega_{2}=0, \Gamma_{2}=\operatorname{Im} \omega_{2}= \pm \alpha_{H} K-P m^{-1} K^{2}
\end{aligned}
$$

Решения (32) указывают на существование неустойчивых колебательных решений для крупномасштабных вихревых возмущений. Максимальный инкремент неустойчивости $\Gamma_{1 \max }=\frac{\alpha_{2}^{2}}{4}$ достигается на волновых числах $K_{\max }=\frac{\alpha_{2}}{2}$. Колебательная мода для магнитных возмущений $\omega_{02}=0$, а максимальный инкремент неустойчивости $\Gamma_{2 \max }=\frac{\alpha_{H}^{2}}{4} P m$ достигает своего значения при волновых числах $K_{\max }=\frac{\alpha_{H}}{2} P m$. Отметим, что в линейной теории, коэффициенты $\alpha_{1}, \alpha_{2}, \alpha_{H}$ не зависят от амплитуд полей, а зависят только от параметра вращения $D$, магнитного числа Прандтля $P m$ и амплитуды внешней силы $f_{0}$. Поскольку коэффициенты $\alpha_{2}, \alpha_{H}$ ответственны за генерацию вихревых и магнитных полей, то проанализируем зависимость этих коэффициентов от безразмерных параметров полагая для простоты безразмерную амплитуду внешней силы $f_{0}$ равной $f_{0}=10$. Фиксирование уровня безразмерной силы означает выбор определенного уровня стационарного фона мелкомасштабных и быстрых осцилляций. Зависимость коэффициентов $\alpha_{2}, \alpha_{H}$ от вращения жидкости (параметра $D$ ) изображена на рис. 1 .
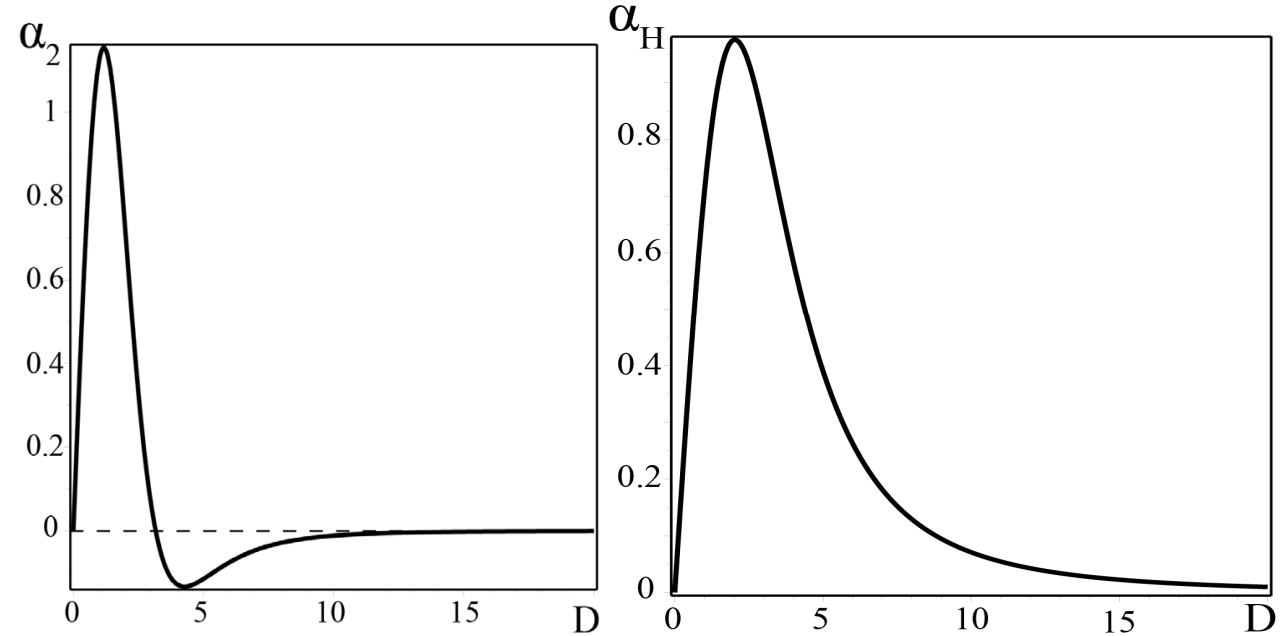

Рис. 1. Слева график зависимости ГД $\alpha$-эффекта от параметра вращения жидкости $D$; справа-график зависимости МГд $\alpha$-эффекта от параметра вращения жидкости $D$ для магнитных чисел Прандтля $P m=1$.

Как видно из рис. 1 , «медленное» вращение жидкости приводит к увеличению $\alpha_{2}$ (гидродинамический $\alpha$ - эффект) до максимального значения $\alpha_{2 \max }$, после которого при дальнейшем увеличении параметра $D$ («быстрое» вращение) наблюдается спад значений $\alpha_{2}$. Аналогичный эффект был впервые описан в работах $[1,19]$. Кроме того при «быстром» вращении жидкости становятся существенными члены порядка $D^{2}$, которые приводят к перемене знака в ГД $\alpha$ - эффекте: $\alpha_{2} \rightarrow-\alpha_{2}$ (рис. 1). В этом случае затухающая мода ГД $\alpha-$ эффекта становится нарастающей. В левой части рис. 3 показана зависимость скорости эволюции крупномасштабных вихревых возмущений $\Gamma_{1}$ относительно волновых чисел $K$ для параметра вращения 
$D=2$. Магнитогидродинамический $\alpha$-эффект (или $\alpha_{H}$-эффект) также увеличивается при «медленном» вращении до максимального значения $\alpha_{\text {Hтах }}$, после которого при увеличении параметра $D$ наблюдается спад $\alpha_{H}$, но знак коэффициента $\alpha_{H}$ не изменяется. Это явление отчетливо прослеживается в правой части рис. 1 для магнитных чисел Прандтля $P m=1$. В случае произвольных значений $P m$ при «быстром» вращении жидкости мы наблюдаем подавление $\alpha_{H}$ - эффекта (см. рис. 2). График скорости эволюции крупномасштабного магнитного поля $\Gamma_{2}$ относительно волновых чисел $K$ для параметра $D=2$ и $P m=1$ показан в правой части рис. 3. В результате развития неустойчивостей во вращающейся электропроводящей жидкости генерируются крупномасштабные спиральные циркулярно-поляризованные вихри и магнитные поля Бельтрамиевского типа.

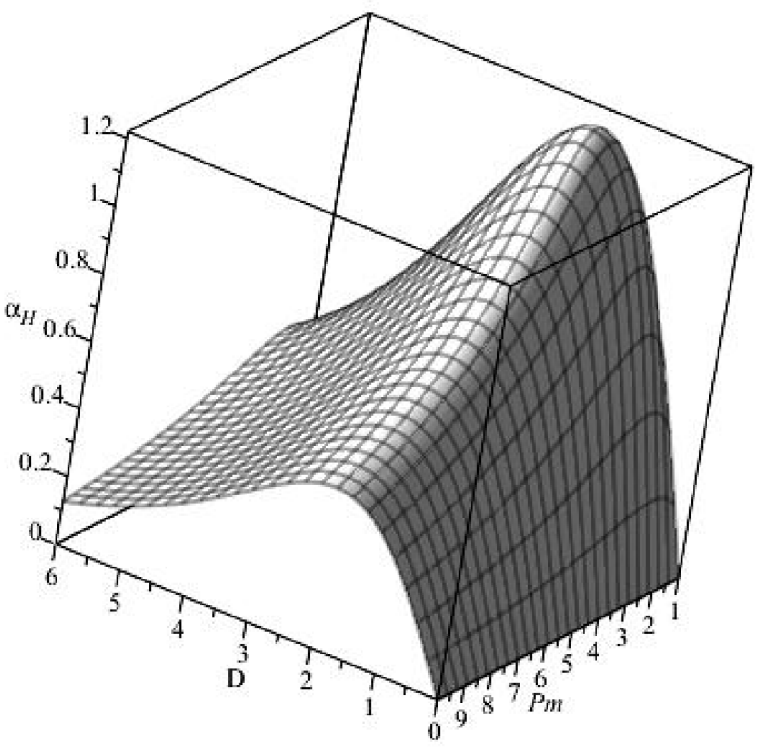

Рис. 2. Трехмерное изображение зависимости коэффициента $\alpha_{H}$ от параметра вращения $D$ и числа Прандтля $P m$.
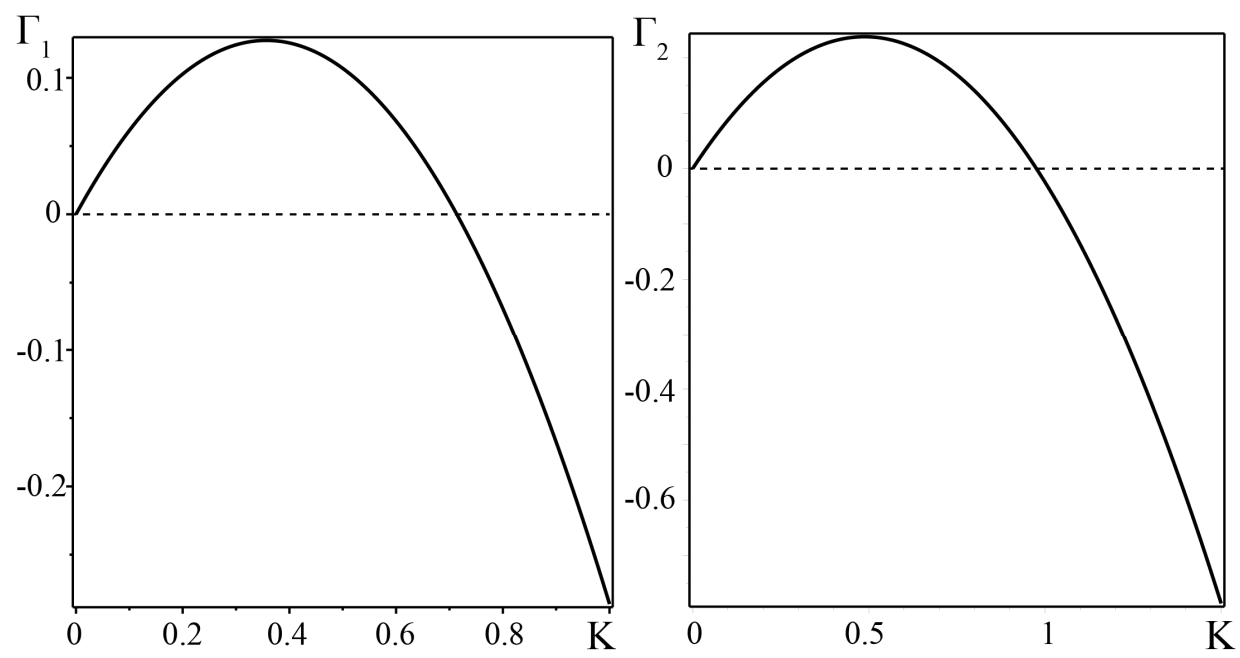

Рис. 3. Слева - график зависимости инкремента неустойчивости для ГД $\alpha$ - эффекта от волновых чисел $K$ при параметре вращения $D=2$, справа - график зависимости инкремента неустойчивости для МГД $\alpha$ - эффекта от волновых чисел $K$ при параметре вращения $D=2$.

\section{НЕЛИНЕЙНЫЕ СТАЦИОНАРНЫЕ СТРУКТУРЫ}

Естественно, что с ростом амплитуды возмущений $W_{1,2}$ и $H_{1,2}$ нелинейные коэффициенты $\alpha_{(1)}, \alpha_{(2)}$, $\alpha_{H}^{(1)}, \alpha_{H}^{(2)}$ уменьшаются и неустойчивость насыщается, переходя к стационарному режиму. В результате образуются нелинейные стационарные структуры. Для описания таких структур рассмотрим нелинейную 
систему уравнений (21)-(24) в стационарном случае, полагая $\partial_{T} W_{1}=\partial_{T} W_{2}=\partial_{T} H_{1}=\partial_{T} H_{2}=0$ и интегрируя эти уравнения по Z:

$$
\begin{aligned}
& \frac{d \widetilde{W}_{1}}{d Z}=-\alpha_{(1)} \widetilde{W}_{2}+C_{1}, \\
& \frac{d \widetilde{W}_{2}}{d Z}=\alpha_{(2)} \widetilde{W}_{1}+C_{2}, \\
& \frac{1}{P m} \frac{d H_{1}}{d Z}=\alpha_{H}^{(2)} H_{2}+C_{3}, \\
& \frac{1}{P m} \frac{d H_{2}}{d Z}=-\alpha_{H}^{(1)} H_{1}+C_{4} .
\end{aligned}
$$

Здесь приняты обозначения $\widetilde{W}_{1}=1-W_{1}, \widetilde{W}_{2}=1-W_{2} ; C_{1}, C_{2}, C_{3}$ и $C_{4}$-произвольные постоянные интегрирования. Общий анализ нелинейной стационарной системы уравнений (34)-(37) представляет собой довольно сложную задачу, выходящую за рамки настоящей работы. Поэтому рассмотрим какие нелинейные структуры могут возникать во вращающейся электропроводящей турбулентной среде в разных физических условиях.

Рассмотрим случай слабой электропроводности среды, когда магнитное число Прандтля и число Чандрасекара малы: $P m \rightarrow 0, Q \rightarrow 0$. Малость магнитных чисел Прандтля $P m$ может быть обусловлена как слабой электропроводностью $(\sigma \rightarrow 0)$ среды, так и очень малой кинематической вязкостью $(v \rightarrow 0)$ среды. При этих условиях генерация крупномасштабного магнитного поля не эффективна $H_{1,2} \ll 1$. В такой среде под воздействием внешней мелкомасштабной силы и силы Кориолиса возможна генерация крупномасштабных вихревых структур бельтрамиевского типа [41]. Стационарные нелинейные вихревые структуры такого типа описываются следующей системой уравнений:

$$
\begin{aligned}
& \frac{d W_{1}}{d Z}=-\frac{f_{0}^{2}}{8} \frac{D^{2}}{16\left(1-W_{1}\right)^{2}+\left[\frac{D^{2}}{2}+4-\left(1-W_{1}\right)^{2}\right]^{2}}+ \\
& +\frac{f_{0}^{2}}{2} \frac{D}{16\left(1-W_{2}\right)^{2}+\left[\frac{D^{2}}{2}+4-\left(1-W_{2}\right)^{2}\right]^{2}}+C_{1} \\
& \frac{d W_{2}}{d Z}=-\frac{f_{0}^{2}}{8} \frac{D^{2}}{16\left(1-W_{2}\right)^{2}+\left[\frac{D^{2}}{2}+4-\left(1-W_{2}\right)^{2}\right]^{2}}- \\
& -\frac{f_{0}^{2}}{2} \frac{D}{16\left(1-W_{1}\right)^{2}+\left[\frac{D^{2}}{2}+4-\left(1-W_{1}\right)^{2}\right]^{2}}+C_{2}
\end{aligned}
$$

Система уравнений (38)-(39) полностью совпадает с аналогичными уравнениями, полученными в обычной гидродинамике [41].

При фиксированных значениях параметров $f_{0}$ и $D$ фазовый портрет динамической системы (38)-(39) определяется значениями постоянных $C_{1}$ и $C_{2}$. Стандартными методами можно доказать, что система уравнений (38)-(39) имеет четыре неподвижные точки в области показанной на рис. 4. Область построена численно при $f_{0}=D=1$. Вне этой области неподвижные точки отсутствуют. На границе реализуются вырожденные случаи - двух неподвижных точек. При небольшом изменении значений параметра, например $D$, область существования четырех неподвижных точек незначительно деформируется и меняет свои размеры и форму. 


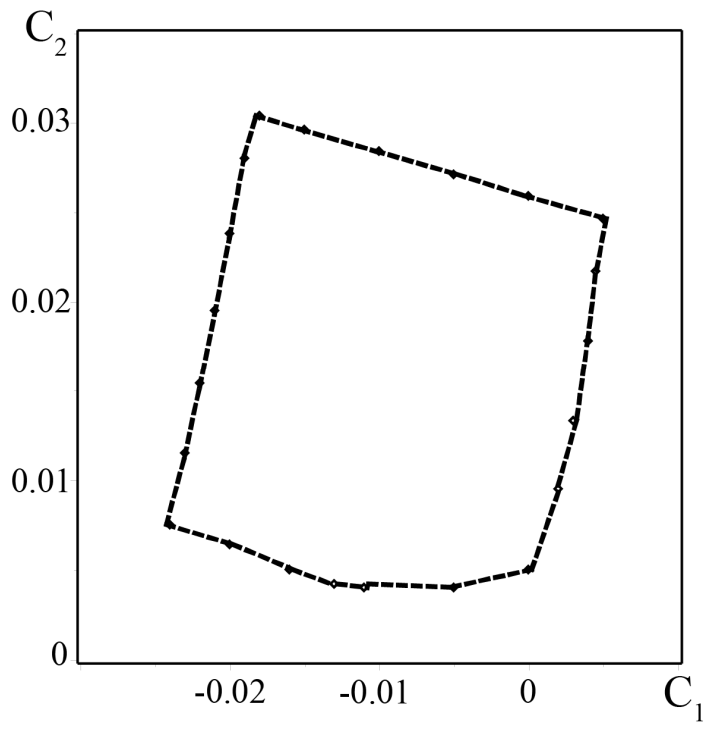

Рис. 4. Граница области параметров на плоскости $\left(C_{1}, C_{2}\right)$ показана штрихованной линией

При значениях параметров принадлежащих штриховой линии на фазовом портрете присутствуют две неподвижные точки. Если параметры лежат внутри этой области появляются четыре неподвижные точки, если вне неподвижные точки отсутствуют

При значениях постоянных $C_{1}$ и $C_{2}$ (рис. 4), когда присутствуют четыре неподвижные точки, линеаризуя векторное поле (38)-(39) в окрестности неподвижных точек установим тип неподвижных точек. Положение и тип неподвижных точек позволяет построить фазовый портрет динамической системы (38)-(39). Знание фазового портрета позволяет качественно описать все возможные стационарные вихревые решения. Типичный фазовый портрет системы уравнений (38)-(39) показан на рис. 5. Наиболее интересные локализованные решения соответствуют траекториям фазового портрета, соединяющим неподвижные (особые) точки на фазовой плоскости. В частности, сепаратриса, соединяющая гиперболическую особую точку с устойчивым фокусом соответствует решению для локализованной вихревой структуры типа кинка с вращением. Это решение, приведенное на рис. 6, получено численным интегрированием. Другой вид спирального кинка соответствует решению, для которого на фазовой плоскости сепаратриса связывает неустойчивый и устойчивый фокус (рис. 6). Перейдем теперь к случаю, когда магнитное число Прандтля отлично от нуля и равно, например, единице: $P m=1$.

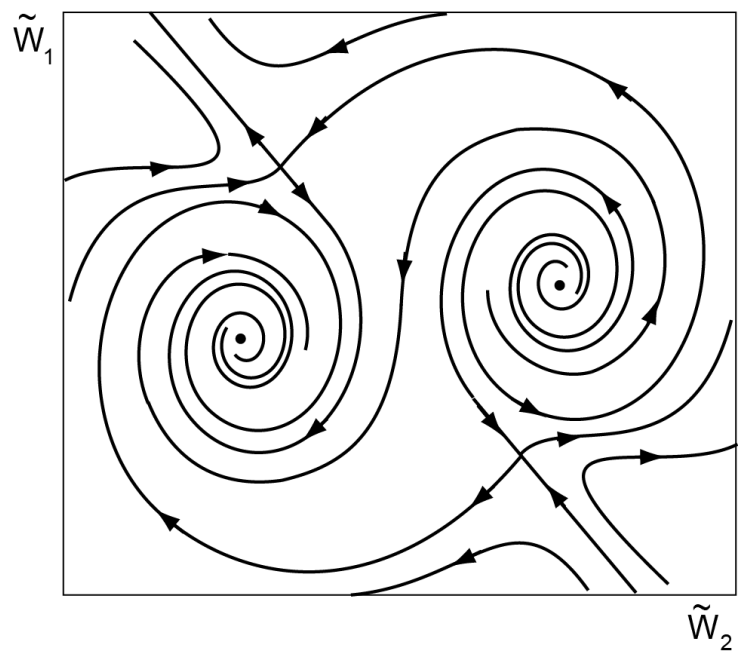

Рис. 5. Фазовый портрет динамической системы (38)-(39) при $f_{0}=D=1, C_{1}=-0,01$ и $C_{2}=0,01$. Видно присутствие двух гиперболических особых точек и устойчивого и неустойчивого фокусов.

В этом случае во вращающейся электропроводящей жидкости под действием внешней мелкомасштабной силы $\vec{F}_{0}$ возникает генерация крупномасштабного магнитного поля, причем максимальная скорость роста магнитных возмущений $\Gamma_{2 \max }$ превышает максимальную скорость роста крупномасштабных вихревых возмущений $\Gamma_{1 \max }$ : 


$$
\gamma^{-1}=\frac{\Gamma_{2 \max }}{\Gamma_{1 \max }}=\left(\frac{\alpha_{H}}{\alpha_{2}}\right)^{2} P m \approx 2,09 \cdot 10^{2}
$$

для чисел $P m=1, D=3$.
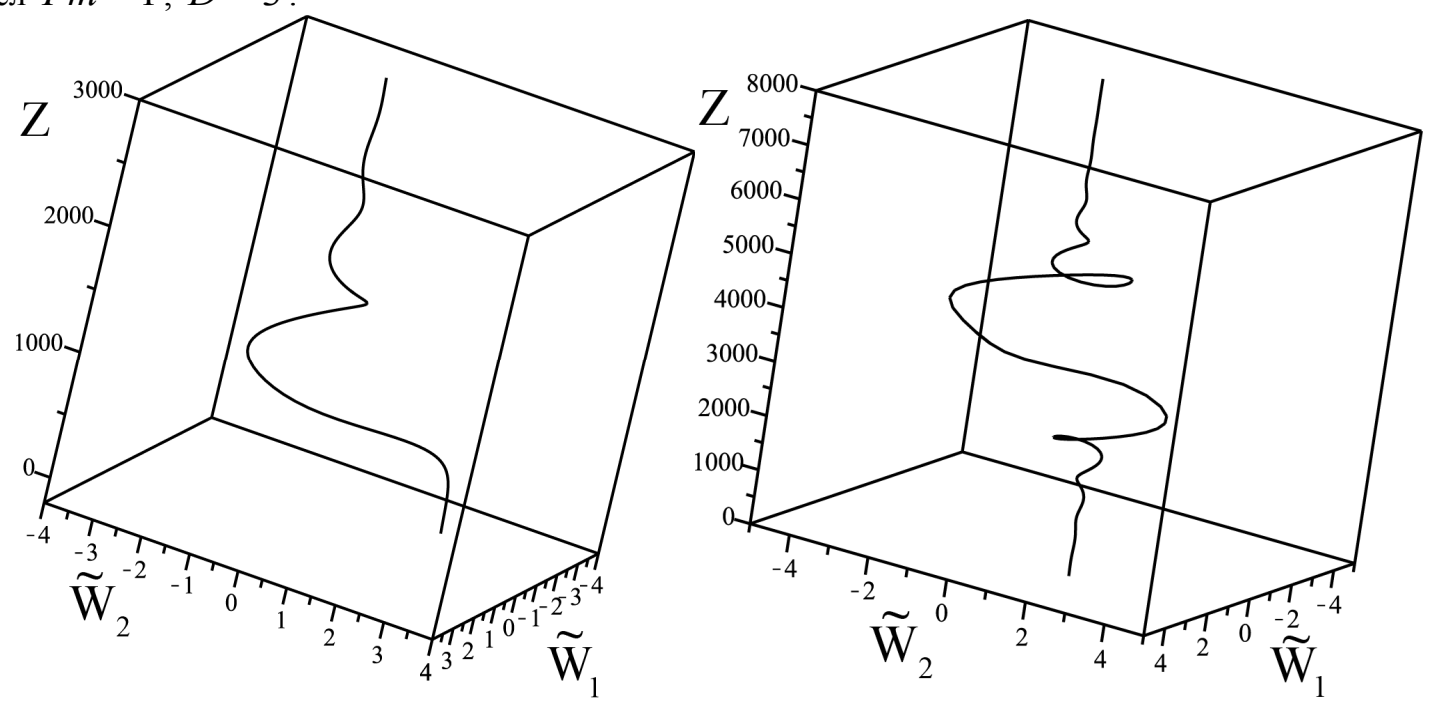

Рис. 6. Численное решение уравнений (38)-(39) для стационарных полей $W_{1,2}$

Слева показан кинк, связывающий гиперболическую точку с устойчивым фокусом; справа - кинк, связывающий неустойчивый и устойчивый фокус.

Исходя из этого обстоятельства, можно представить следующую картину протекающих физических процессов: генерируемое крупномасштабное вихревое возмущение во вращающейся среде еще не вышло на стационарный режим, а возмущения крупномасштабного магнитного поля уже достигли своего насыщения на стационарном уровне. Очевидно, что влиянием малых амплитуд крупномасштабных вихревых возмущений $W_{1,2} \ll 1$ на эволюцию крупномасштабного магнитного поля мы можем пренебречь. В результате стационарное крупномасштабное магнитное поле будет описываться следующей системой уравнений:

$$
\begin{aligned}
& \frac{d \tilde{H}_{1}}{d Z}=f_{0}^{2} \frac{D\left(5-\tilde{H}_{2}^{2}\right) \tilde{H}_{2}}{\tilde{H}_{2}^{8}+20 \tilde{H}_{2}^{6}+a \tilde{H}_{2}^{4}+b \tilde{H}_{2}^{2}+c}+C_{3}^{\prime}, \\
& \frac{d \tilde{H}_{2}}{d Z}=-f_{0}^{2} \frac{D\left(5-\tilde{H}_{1}^{2}\right) \tilde{H}_{1}}{\tilde{H}_{1}^{8}+20 \tilde{H}_{1}^{6}+a \tilde{H}_{1}^{4}+b \tilde{H}_{1}^{2}+c}+C_{4}^{\prime},
\end{aligned}
$$

где введены обозначения: $\quad \tilde{H}_{1,2}=H_{1,2} \sqrt{Q}, \quad C_{3,4}^{\prime}=C_{3,4} \sqrt{Q}, \quad a=3 D^{2}+150, \quad b=50\left(D^{2}+10\right)$, $c=25\left(\left(\frac{D^{2}}{2}+3\right)^{2}+16\right)$.

Приступим к исследованию вопроса о видах стационарных магнитных структур, описываемых системой уравнений (41)-(42). Как и в предыдущем случае, установим область изменения постоянных $\left(C_{3}, C_{4}\right)$, которая определяется путем вычисления максимума и минимума функции

$$
\Phi_{1,2}=f_{0}^{2} \frac{D\left(5-\tilde{H}_{1,2}^{2}\right) \tilde{H}_{1,2}}{\tilde{H}_{1,2}^{8}+20 \tilde{H}_{1,2}^{6}+a \tilde{H}_{1,2}^{4}+b \tilde{H}_{1,2}^{2}+c} .
$$

Обозначая через $\chi=\max \left(f_{0}^{2} \frac{D\left(5-\widetilde{H}_{2}^{2}\right) \widetilde{H}_{2}}{\widetilde{H}_{2}^{8}+20 \widetilde{H}_{2}^{6}+a \widetilde{H}_{2}^{4}+b \widetilde{H}_{2}^{2}+c}\right)$ при изменении $\widetilde{H}_{2}$, область изменения постоянных $\left(C_{3}, C_{4}\right)$ определяется следующими неравенствами

$$
\begin{aligned}
& -\chi<C_{3}<\chi \\
& -\chi<C_{4}<\chi
\end{aligned}
$$




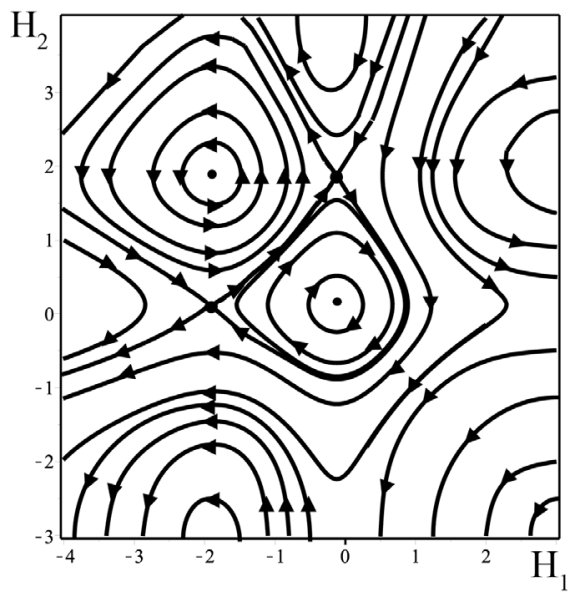

Рис. 7. Фазовый портрет динамической системы уравнений (41)-(42) при $f_{0}=Q=1, D=3, C_{1}=-0,001$ и $C_{2}=-0,001$.

Видно присутствие двух эллиптических и двух гиперболических особых точек.

Для фиксированных значений $f_{0}, D, Q$ этот максимум легко вычисляется. Далее при выполнении всех численных расчетов примем $f_{0}=1, D=3, Q=1$. Линеаризуя правые части системы уравнений (41)-(42) в окрестности неподвижных точек мы можем установить их тип и построить соответствующий фазовый портрет. Так при выборе постоянных $C_{3} \neq 0\left(C_{3}=-0,001\right)$ и $C_{4} \neq 0\left(C_{4}=-0,001\right)$ мы получаем четыре неподвижные точки: две эллиптического и две гиперболического типов. Фазовый портрет для этого случая $\left(C_{3} \neq 0\right.$ и $C_{4} \neq 0$ ) представлен на рис. 7. Из него видно существование двух типов ограниченных стационарных решений, это нелинейные волны и кинки. Нелинейным волнам соответствуют замкнутые траектории фазового портрета, а кинкам сепаратрисы, соединяющие гиперболические точки (рис. 8). В принципе нелинейные волны можно разделить на два типа, в зависимости от неподвижной эллиптической точки, которую они окружают. Аналогично в этом случае можно различать и два типа кинков по сегментам сепаратрис, соединяющих две гиперболические точки.
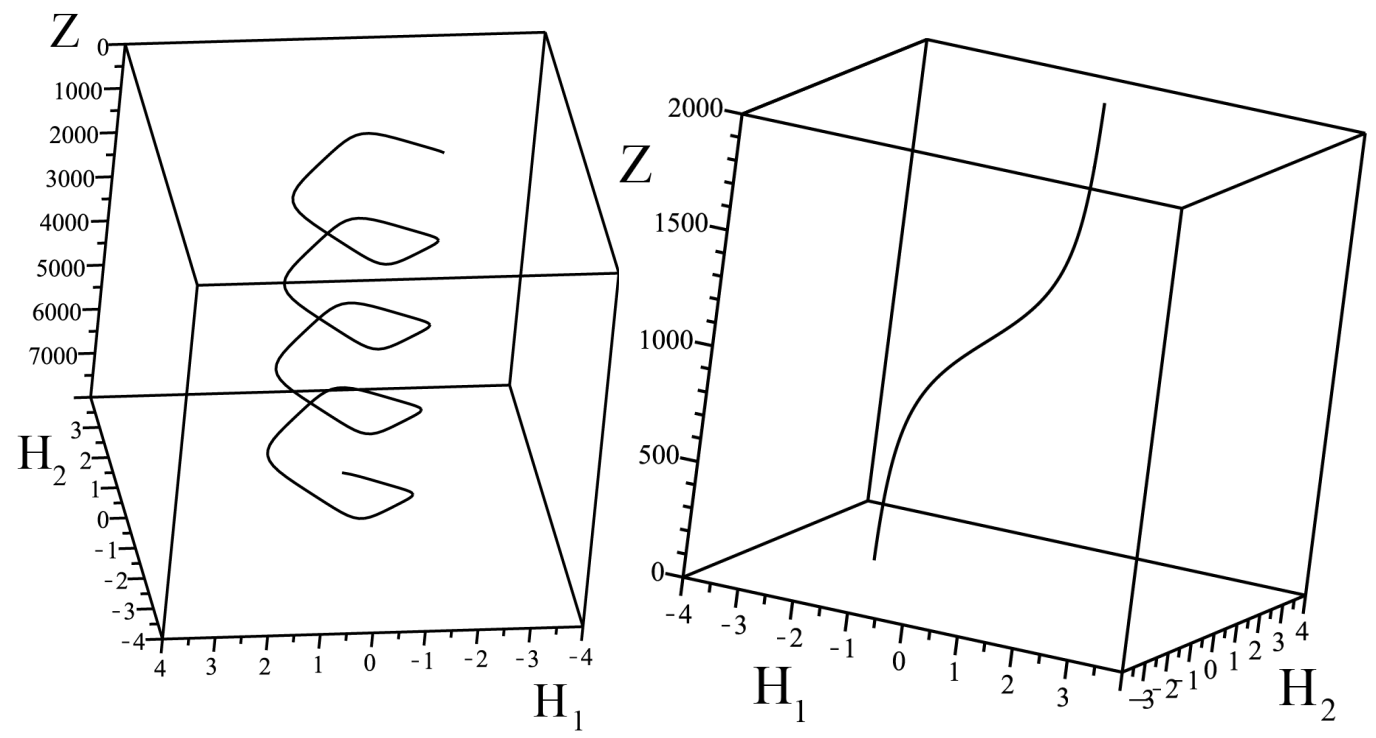

Рис. 8. Численное решение уравнений (41)-(42) для стационарных магнитных полей $H_{1,2}$

Слева изображено стационарное решение в окрестности эллиптической точки, соответствующее нелинейной волне; справастационарное решение, соответствующее кинку.

\section{ЗАКЛЮЧЕНИЕ}

В работе получена замкнутая система нелинейных уравнений для вихревых и магнитных крупномасштабных возмущений (магнито-вихревое динамо), описывающую как линейную, так и нелинейную стадии нарастания этих возмущений в электропроводящей среде со внешней мелкомасштабной силой. Эта сила поддерживает малые флуктуации поля скорости и создает мелкомасштабную турбулентность с малым числом Рейнольдса $R \ll 1$. Асимптотическое разложение уравнений движения по малому числу Рейнольдса позволяет 
получить уравнения движения для больших масштабов. Показано, что на линейной стадии генерация крупномасштабных вихревых возмущений происходит благодаря развитию неустойчивости типа гидродинамического $\alpha$-эффекта, а генерация крупномасштабных магнитных полей из-за неустойчивости типа МГД $\alpha$-эффекта. Неустойчивость обусловлена совместным действием мелкомасштабной внешней силы и силы Кориолиса. С ростом амплитуды вихревых и магнитных возмущений неустойчивость стабилизируется и переходит в стационарный режим. В случае, когда электропроводность среды очень мала или параметр вращения мал, то нелинейные уравнения магнито-вихревого динамо трансформируются в вихревую подсистему уравнений. В этом режиме формируются нелинейные стационарные вихревые структурыспиральные кинки, аналогичные тем, которые были найдены в обычной гидродинамике в работе [41]. Если электропроводность среды существенна, а вихревые возмущения малы, то для магнитной подсистемы мы получили решения в виде периодических нелинейных волн и кинков. Проведенный в работе качественный анализ системы уравнений (38)-(39) и (41)-(42) был выполнен для фиксированных значений параметров турбулентности $f_{0}$, вращения $D$, магнитного поля $Q$ и констант $C_{1,2,3,4}$. Для более полного описания физической природы, рассматриваемых здесь нелинейных стационарных структур, естественно исследовать изменения общего фазового портрета системы уравнений (34)-(37) при изменение параметров системы. Такой бифуркационный качественный анализ этой многопараметрической задачи выглядит исключительно громоздким и сложным и требует специального рассмотрения, выходящего за рамки этой работы. 
ПРИЛОЖЕНИЕ I.

\section{МНОГОМАСШТАБНЫЕ АСИМПТОТИЧЕСКИЕ РАЗЛОЖЕНИЯ}

Приведем алгебраическую структуру асимптотического разложения уравнений (7)-(9) в различных порядках по $R$, начиная с наименьшего. В порядке $R^{-3}$ имеется только одно уравнение:

$$
\partial_{i} P_{-3}=0 \Rightarrow P_{-3}=P_{-3}(X)
$$

В порядке $R^{-2}$ имеем уравнение:

$$
\partial_{i} P_{-2}=0 \Rightarrow P_{-2}=P_{-2}(X)
$$

Уравнения (43) и (44) выполняются автоматически поскольку $P_{-3}$ и $P_{-2}$ являются функциями только медленных переменных.

В порядке $R^{-1}$ получаем систему уравнений:

$$
\begin{aligned}
& \partial_{t} W_{-1}^{i}+W_{-1}^{k} \partial_{k} W_{-1}^{i}=-\partial_{i} P_{-1}-\nabla_{i} P_{-3}+\partial_{k}^{2} W_{-1}^{i}+\varepsilon_{i j k} W_{j} D_{k}+\tilde{Q} \varepsilon_{i j k} \varepsilon_{j m l} \partial_{m} B_{-1}^{l} B_{-1}^{k} \\
& \partial_{t} B_{-1}^{i}-P m^{-1} \partial_{k}^{2} B_{-1}^{i}=\varepsilon_{i j k} \varepsilon_{k n p} \partial_{j} W_{-1}^{n} B_{-1}^{p} \\
& \partial_{i} W_{-1}^{i}=0, \quad \partial_{i} B_{-1}^{i}=0
\end{aligned}
$$

Усреднение уравнений (45) по «быстрым» переменным дает секулярное уравнение:

$$
-\nabla_{i} P_{-3}+\varepsilon_{i j k} W_{j} D_{k}=0
$$

которое соответствует геострофическому равновесию.

В нулевом порядке по $R$ имеем следующую систему уравнений:

$$
\begin{aligned}
& \partial_{t} v_{0}^{i}+W_{-1}^{k} \partial_{k} v_{0}^{i}+v_{0}^{k} \partial_{k} W_{-1}^{i}=-\partial_{i} P_{0}-\nabla_{i} P_{-2}+\partial_{k}^{2} v_{0}^{i}+\varepsilon_{i j k} v_{0}^{j} D_{k}+\tilde{Q} \varepsilon_{i j k} \varepsilon_{j m l}\left(\partial_{m} B_{-1}^{l} B_{0}^{k}+\partial_{m} B_{0}^{l} B_{-1}^{k}\right)+F_{0}^{i} \\
& \partial_{t} B_{0}^{i}-P m^{-1} \partial_{k}^{2} B_{0}^{i}=\varepsilon_{i j k} \varepsilon_{k n p}\left(\partial_{j} W_{-1}^{n} B_{0}^{p}+\partial_{j} v_{0}^{n} B_{-1}^{p}\right) \\
& \partial_{i} v_{0}^{i}=0, \quad \partial_{i} B_{0}^{i}=0
\end{aligned}
$$

Эти уравнения дают один секулярный член:

$$
\nabla P_{-2}=0 \Rightarrow P_{-2}=\text { const }
$$

Рассмотрим приближение первого порядка $R^{1}$ :

$$
\begin{gathered}
\partial_{t} v_{1}^{i}+W_{-1}^{k} \partial_{k} v_{1}^{i}+v_{0}^{k} \partial_{k} v_{0}^{i}+v_{1}^{k} \partial_{k} W_{-1}^{i}+W_{-1}^{k} \nabla_{k} W_{-1}^{i}=-\nabla_{i} P_{-1}- \\
-\partial_{i}\left(P_{1}+\bar{P}_{1}\right)+\partial_{k}^{2} v_{1}^{i}+2 \partial_{k} \nabla_{k} W_{-1}^{i}+\varepsilon_{i j k} v_{1}^{j} D_{k}+ \\
+\tilde{Q} \varepsilon_{i j k} \varepsilon_{j m l}\left(\partial_{m} B_{-1}^{l} B_{1}^{k}+\partial_{m} B_{0}^{l} B_{0}^{k}+\partial_{m} B_{1}^{l} B_{(-1)}^{k}+\nabla_{m} B_{(-1)}^{l} B_{(-1)}^{k}\right) \\
\partial_{t} B_{1}^{i}-P m^{-1} \partial_{k}^{2} B_{1}^{i}-P m^{-1} \partial_{k} \nabla_{k} B_{-1}^{i}=\varepsilon_{i j k} \varepsilon_{k n p}\left(\partial_{j} W_{-1}^{n} B_{1}^{p}+\partial_{j} v_{0}^{n} B_{0}^{p}+\partial_{j} v_{1}^{n} B_{-1}^{p}+\nabla_{j} W_{-1}^{n} B_{-1}^{p}\right) \\
\partial_{i} v_{1}^{i}+\nabla_{i} W_{-1}^{i}=0, \quad \partial_{i} B_{1}^{i}+\nabla_{i} B_{-1}^{i}=0
\end{gathered}
$$

Из этой системы уравнений следуют секулярные уравнения

$$
\begin{gathered}
W_{-1}^{k} \nabla_{k} W_{-1}^{i}=-\nabla_{i} P_{-1}+\tilde{Q} \varepsilon_{i j k} \varepsilon_{j m l} \nabla_{m} B_{-1}^{l} B_{-1}^{k} \\
\varepsilon_{i j k} \varepsilon_{k n p} \nabla_{j} W_{-1}^{n} B_{-1}^{p}=0 \\
\nabla_{i} W_{-1}^{i}=0 \quad \nabla_{i} B_{-1}^{i}=0
\end{gathered}
$$

Для второго порядка $R^{2}$ получим уравнения: 


$$
\begin{array}{r}
\partial_{t} v_{2}^{i}+W_{-1}^{k} \partial_{k} v_{2}^{i}+v_{0}^{k} \partial_{k} v_{1}^{i}+W_{-1}^{k} \nabla_{k} v_{0}^{i}+v_{0}^{k} \nabla_{k} W_{-1}^{i}+v_{1}^{k} \partial_{k} v_{0}^{i}+v_{2}^{k} \partial_{k} W_{-1}^{i}= \\
=-\nabla_{i} P_{2}-\nabla_{i} P_{0}+\partial_{k}^{2} v_{2}^{i}+2 \partial_{k} \nabla_{k} v_{0}^{i}+\varepsilon_{i j k} v_{2}^{j} D_{k}+ \\
+\tilde{Q} \varepsilon_{i j k} \varepsilon_{j m l}\left(\partial_{m} B_{-1}^{l} B_{2}^{k}+\partial_{m} B_{0}^{l} B_{1}^{k}+\partial_{m} B_{1}^{l} B_{0}^{k}+\partial_{m} B_{2}^{l} B_{-1}^{k}+\nabla_{m} B_{-1}^{l} B_{0}^{k}+\nabla_{m} B_{0}^{l} B_{-1}^{k}\right) \\
\partial_{t} B_{2}^{i}-P m^{-1} \partial_{k}^{2} B_{2}^{i}-P m^{-1} 2 \partial_{k} \nabla_{k} B_{0}^{i}=\varepsilon_{i j k} \varepsilon_{k n p}\left(\partial_{j} W_{-1}^{n} B_{2}^{p}+\partial_{j} v_{0}^{n} B_{1}^{p}+\right. \\
\left.+\partial_{j} v_{2}^{n} B_{-1}^{p}+\nabla_{j} W_{-1}^{n} B_{0}^{p}+\nabla_{j} v_{0}^{n} B_{-1}^{p}\right) \\
\partial_{i} v_{2}^{i}+\nabla_{i} v_{0}^{i}=0, \quad \partial_{i} B_{2}^{i}+\nabla_{i} B_{0}^{i}=0
\end{array}
$$

После усреднения системы уравнений (52) по быстрым переменным видно, что в порядке $R^{2}$ секулярных членов нет.

Наконец приходим к наиболее важному порядку $R^{3}$. В этом порядке уравнении имеют следующий вид:

$$
\begin{gathered}
\partial_{t} v_{3}^{i}+\partial_{T} W_{-1}^{i}+W_{-1}^{k} \partial_{k} v_{3}^{i}+v_{0}^{k} \partial_{k} v_{2}^{i}+W_{-1}^{k} \nabla_{k} v_{1}^{i}+v_{0}^{k} \nabla_{k} v_{0}^{i}+v_{1}^{k} \partial_{k} v_{1}^{i}+v_{1}^{k} \nabla_{k} W_{-1}^{i}+v_{2}^{k} \partial_{k} W_{-1}^{i}= \\
=-\partial_{i} P_{3}-\nabla_{i}\left(P_{1}+\bar{P}_{1}\right)+\partial_{k}^{2} v_{3}^{i}+2 \partial_{k} \nabla_{k} v_{1}^{i}+\Delta W_{-1}^{i}+\varepsilon_{i j k} v_{3}^{j} D_{k}+ \\
+\tilde{Q} \varepsilon_{i j k} \varepsilon_{j m l}\left(\partial_{m} B_{-1}^{l} B_{3}^{k}+\partial_{m} B_{0}^{l} B_{2}^{k}+\partial_{m} B_{1}^{l} B_{1}^{k}+\partial_{m} B_{2}^{l} B_{0}^{k}+\nabla_{m} B_{-1}^{l} B_{1}^{k}+\nabla_{m} B_{0}^{l} B_{0}^{k}\right), \\
\partial_{t} B_{3}^{i}+\partial_{T} B_{-1}^{i}-P m^{-1} \partial_{k}^{2} B_{3}^{i}-P m^{-1} 2 \partial_{k} \nabla_{k} B_{1}^{i}-P m^{-1} \Delta B_{-1}^{i}= \\
=\varepsilon_{i j k} \varepsilon_{k n p}\left(\partial_{j} W_{-1}^{n} B_{3}^{p}+\partial_{j} v_{0}^{n} B_{2}^{p}+\partial_{j} v_{1}^{n} B_{1}^{p}+\partial_{j} v_{2}^{n} B_{0}^{p}+\nabla_{j} W_{-1}^{n} B_{1}^{p}+\nabla_{j} v_{0}^{n} B_{0}^{p}\right), \\
\partial_{i} v_{3}^{i}+\nabla_{i} v_{1}^{i}=0, \quad \partial_{i} B_{3}^{i}+\nabla_{i} B_{1}^{i}=0 .
\end{gathered}
$$

Усредняя эту систему уравнений по быстрым переменным получим основные секулярные уравнения, описывающие эволюцию крупномасштабных возмущений:

$$
\begin{gathered}
\partial_{t} W_{-1}^{i}-\Delta W_{-1}^{i}+\nabla_{k}\left(\overline{v_{0}^{k} v_{0}^{i}}\right)=-\nabla_{i} \bar{P}_{1}+\tilde{Q} \varepsilon_{i j k} \varepsilon_{j m l} \nabla_{m}\left(\overline{B_{0}^{l} B_{0}^{k}}\right), \\
\partial_{t} B_{-1}^{i}-P m^{-1} \Delta B_{-1}^{i}=\varepsilon_{i j k} \varepsilon_{k n p} \nabla_{j}\left(\overline{v_{0}^{n} B_{0}^{p}}\right) .
\end{gathered}
$$


ПРИЛОЖЕНИЕ II.

\section{МЕЛКОМАСШТАБНЫЕ ПОЛЯ В НУЛЕВОМ ПОРЯДКЕ ПО $R$}

В Приложении I мы получили уравнения асимптотического разложения в нулевом приближении, которые можно записать в следующем виде:

$$
\begin{gathered}
\widehat{D}_{W} v_{0}^{i}=-\partial_{i} P_{0}+\varepsilon_{i j k} v_{0}^{j} D_{k}+\widehat{Q} H_{k}\left(\partial_{k} B_{0}^{i}-\partial_{i} B_{0}^{k}\right)+F_{0}^{i}, \\
\widehat{D}_{H} B_{0}^{i}=\left(H_{p} \partial_{p}\right) v_{0}^{i}, \\
\partial_{i} v_{0}^{i}=\partial_{k} B_{0}^{k}=0,
\end{gathered}
$$

где введены обозначения для операторов:

$$
\hat{D}_{W}=\partial_{t}-\partial_{k}^{2}+W_{-1}^{k} \partial_{k}, \hat{D}_{H}=\partial_{t}-P m^{-1} \partial_{k}^{2}+W_{-1}^{k} \partial_{k}
$$

Мелкомасштабные осцилляции магнитного поля легко находятся из уравнения (26):

$$
B_{0}^{i}=\frac{\left(H_{p} \partial_{p}\right)}{\hat{D}_{H}} v_{0}^{i}
$$

Подставим (59) в (56), и воспользовавшись условием соленоидальности полей $\vec{v}_{0}$ и $\vec{B}_{0}(58)$, находим давление $P_{0}$ :

$$
P_{0}=\hat{P}_{1} u_{0}+\hat{P}_{2} v_{0}+\hat{P}_{3} w_{0}
$$

Здесь мы ввели обозначения для операторов:

$$
\hat{P}_{1}=\frac{D_{2} \partial_{z}-D_{3} \partial_{y}}{\partial^{2}}-\tilde{Q} H_{1} \frac{\left(H_{p} \partial_{p}\right)}{\hat{D}_{H}}, \quad \hat{P}_{2}=\frac{D_{3} \partial_{x}-D_{1} \partial_{z}}{\partial^{2}}-\tilde{Q} H_{2} \frac{\left(H_{p} \partial_{p}\right)}{\hat{D}_{H}}, \quad P_{3}=\frac{D_{1} \partial_{y}-D_{2} \partial_{x}}{\partial^{2}}
$$

и скоростей: $v_{0}^{x}=u_{0}, v_{0}^{y}=v_{0}, v_{0}^{z}=w_{0}$. Используя представление (60), мы можем исключить давление из уравнения (56) и в результате получить систему уравнений для нахождения поля скорости нулевого приближения:

$$
\begin{aligned}
& \left(D_{3}+\hat{p}_{1 y}\right) u_{0}+\left(\widehat{D}_{W}-\frac{\widetilde{Q}\left(H_{p} \partial_{p}\right)^{2}}{\widehat{D}_{H}}+\hat{p}_{2 y}\right) v_{0}+\left(\hat{p}_{3 y}-D_{1}\right) w_{0}=F_{0}^{y} \\
& \left(\widehat{D}_{W}-\frac{\widetilde{Q}\left(H_{p} \partial_{p}\right)^{2}}{\widehat{D}_{H}}+\hat{p}_{1 x}\right) u_{0}+\left(\hat{p}_{2 x}-D_{3}\right) v_{0}+\left(\hat{p}_{3 x}+D_{2}\right) w_{0}=F_{0}^{x} \\
& \left(\hat{p}_{1 z}-D_{2}\right) u_{0}+\left(\hat{p}_{2 z}+D_{1}\right) v_{0}+\left(\widehat{D}_{W}-\frac{\widetilde{Q}\left(H_{p} \partial_{p}\right)^{2}}{\widehat{D}_{H}}+\hat{p}_{3 z}\right) w_{0}=F_{0}^{z}
\end{aligned}
$$

Компоненты тензора $\hat{p}_{i j}$ имеют следующий вид:

$$
\begin{aligned}
& \hat{p}_{1 x}=\frac{D_{2} \partial_{x} \partial_{z}-D_{3} \partial_{x} \partial_{y}}{\partial^{2}}, \quad \hat{p}_{2 x}=\frac{D_{3} \partial_{x}^{2}-D_{1} \partial_{x} \partial_{z}}{\partial^{2}}, \quad \hat{p}_{3 x}=\frac{D_{1} \partial_{x} \partial_{y}-D_{2} \partial_{x}^{2}}{\partial^{2}}, \\
& \hat{p}_{1 y}=\frac{D_{2} \partial_{y} \partial_{z}-D_{3} \partial_{y}^{2}}{\partial^{2}}, \quad \hat{p}_{2 y}=\frac{D_{3} \partial_{y} \partial_{x}-D_{1} \partial_{y} \partial_{z}}{\partial^{2}}, \quad \hat{p}_{3 y}=\frac{D_{1} \partial_{y}^{2}-D_{2} \partial_{y} \partial_{x}}{\partial^{2}}, \\
& \hat{p}_{1 z}=\frac{D_{2} \partial_{z}^{2}-D_{3} \partial_{z} \partial_{y}}{\partial^{2}}, \quad \hat{p}_{2 z}=\frac{D_{3} \partial_{z} \partial_{x}-D_{1} \partial_{z}^{2}}{\partial^{2}}, \quad \hat{p}_{3 z}=\frac{D_{1} \partial_{z} \partial_{y}-D_{2} \partial_{z} \partial_{x}}{\partial^{2}} .
\end{aligned}
$$


Систему уравнений (62) можно значительно упростить, если выбрать систему координат так, чтобы ось $Z$ совпадала с направлением угловой скорости вращения $\vec{\Omega}$ жидкости. Тогда $D_{1}=0, D_{2}=0, D_{3}=D$. Для решения этой системы уравнений зададим внешнюю силу в явном виде. Для простоты выберем силу $\vec{F}_{0}$ в следующем виде:

$$
\vec{F}_{0}=f_{0}\left(\vec{i} \cos \varphi_{2}+\vec{j} \cos \varphi_{1}\right),
$$

где $f_{0}$ - амплитуда внешней силы, $\phi_{1}=\vec{\kappa}_{1} \vec{x}-\omega_{0} t, \phi_{2}=\vec{\kappa}_{2} \vec{x}-\omega_{0} t, \kappa_{1}=\kappa_{0}(1,0,1), \kappa_{2}=\kappa_{0}(0,1,1)$. Отсюда видно, что сила $\vec{F}_{0}$ задана в плоскости $(x, y)$, ортогональной оси вращения и удовлетворяет свойствам (5). Решение упрощенной системы уравнений (62) находится по правилам Крамера:

$$
\begin{aligned}
& u_{0}=\frac{1}{\Delta}\left\{\left(\hat{D}_{W}-\tilde{Q} \frac{\left(H_{p} \partial_{p}\right)^{2}}{D_{H}}+D \frac{\partial_{y} \partial_{x}}{\partial^{2}}\right)\left(\hat{D}_{W}-\frac{\tilde{Q}\left(H_{p} \partial_{p}\right)^{2}}{D_{H}}\right) F_{0}^{x}-\right. \\
& \left.-\left(D \frac{\partial_{x}^{2}}{\partial^{2}}-D\right)\left(\hat{D}_{W}-\frac{\tilde{Q}\left(H_{p} \partial_{p}\right)^{2}}{\hat{D}_{H}}\right) F_{0}^{y}\right\} \\
& v_{0}=\frac{1}{\Delta}\left\{\left(\hat{D}_{W}-\tilde{Q} \frac{\left(H_{p} \partial_{p}\right)^{2}}{\hat{D}_{H}}-D \frac{\partial_{y} \partial_{x}}{\partial^{2}}\right)\left(\hat{D}_{W}-\frac{\tilde{Q}\left(H_{p} \partial_{p}\right)^{2}}{\hat{D}_{H}}\right) F_{0}^{y}\right. \\
& \left.+\left(D \frac{\partial_{y}^{2}}{\partial^{2}}-D\right)\left(\hat{D}_{W}-\frac{\tilde{Q}\left(H_{p} \partial_{p}\right)^{2}}{\hat{D}_{H}}\right) F_{0}^{x}\right\} \\
& w_{0}=\frac{1}{\Delta}\left\{\left[\left(D-D \frac{\partial_{y}^{2}}{\partial^{2}}\right) D \frac{\partial_{z} \partial_{x}}{\partial^{2}}+D \frac{\partial_{y} \partial_{z}}{\partial^{2}}\left(\hat{D}_{W}-\tilde{Q} \frac{\left(H_{p} \partial_{p}\right)^{2}}{\hat{D}_{H}}+D \frac{\partial_{y} \partial_{x}}{\partial^{2}}\right)\right] F_{0}^{x}+\right. \\
& \left.+\left[\left(D-D \frac{\partial_{x}^{2}}{\partial^{2}}\right) D \frac{\partial_{z} \partial_{y}}{\partial^{2}}-D \frac{\partial_{x} \partial_{z}}{\partial^{2}}\left(\hat{D}_{W}-\tilde{Q} \frac{\left(H_{p} \partial_{p}\right)^{2}}{\hat{D}_{H}}-D \frac{\partial_{y} \partial_{x}}{\partial^{2}}\right)\right] F_{0}^{y}\right\} .
\end{aligned}
$$

Здесь $\Delta$ - детерминант упрощенной системы (62), который в раскрытом виде имеет вид:

$$
\begin{gathered}
\Delta=\left(\hat{D}_{W}-\tilde{Q} \frac{\left(H_{p} \partial_{p}\right)^{2}}{\hat{D}_{H}}-D \frac{\partial_{y} \partial_{x}}{\partial^{2}}\right)\left(\hat{D}_{W}-\tilde{Q} \frac{\left(H_{p} \partial_{p}\right)^{2}}{\hat{D}_{H}}+D \frac{\partial_{y} \partial_{x}}{\partial^{2}}\right)\left(\hat{D}_{W}-\frac{\tilde{Q}\left(H_{p} \partial_{p}\right)^{2}}{\hat{D}_{H}}\right)- \\
-\left(D-D \frac{\partial_{y}^{2}}{\partial^{2}}\right)\left(D \frac{\partial_{x}^{2}}{\partial^{2}}-D\right)\left(\hat{D}_{W}-\frac{\tilde{Q}\left(H_{p} \partial_{p}\right)^{2}}{\hat{D}_{H}}\right) .
\end{gathered}
$$

Для вычисления выражений (65)-(68) запишем внешнюю силу (33) в комплексной форме:

$$
\vec{F}_{0}=\vec{i} \frac{f_{0}}{2} e^{i \varphi_{2}}+\vec{j} \frac{f_{0}}{2} e^{i \varphi_{1}}+\text { c.c. }
$$

Тогда все операторы в формулах (65-68) действуют слева на собственные функции:

$$
\begin{gathered}
\hat{D}_{W, H} e^{i \varphi_{1}}=e^{i \varphi_{1}} \hat{D}_{W, H}\left(\vec{\kappa}_{1},-\omega_{0}\right), \hat{D}_{W, H} e^{i \varphi_{2}}=e^{i \varphi_{2}} \hat{D}_{W, H}\left(\vec{\kappa}_{2},-\omega_{0}\right), \\
\Delta e^{i \varphi_{1}}=e^{i \varphi_{1}} \Delta\left(\vec{\kappa}_{1},-\omega_{0}\right), \Delta e^{i \varphi_{2}}=e^{i \varphi_{2}} \Delta\left(\vec{\kappa}_{2},-\omega_{0}\right) .
\end{gathered}
$$

Полагая $\kappa_{0}=1, \omega_{0}=1$ обозначим: 


$$
\begin{gathered}
\hat{D}_{W}\left(\vec{\kappa}_{1},-\omega_{0}\right)=\hat{D}_{W_{1}}^{*}=2-i\left(1-W_{1}\right), \hat{D}_{W}\left(\vec{\kappa}_{2},-\omega_{0}\right)=\hat{D}_{W_{2}}^{*}=2-i\left(1-W_{2}\right), \\
W_{-1}^{x}=W_{1}, \quad W_{-1}^{y}=W_{2}, \\
\hat{D}_{H}\left(\vec{\kappa}_{1},-\omega_{0}\right)=\hat{D}_{H_{1}}^{*}=2 P m^{-1}-i\left(1-W_{1}\right), \\
\hat{D}_{H}\left(\vec{\kappa}_{2},-\omega_{0}\right)=\hat{D}_{H_{2}}^{*}=2 P m^{-1}-i\left(1-W_{2}\right) .
\end{gathered}
$$

Комплексно-сопряженные величины здесь и далее будем обозначать звездочкой. При выполнении дальнейших вычислений, часть компонент в тензорах $\hat{p}_{i j}\left(\vec{\kappa}_{1}\right)$ и $\hat{p}_{i j}\left(\vec{\kappa}_{2}\right)$ обращается в ноль, поэтому выпишем только не нулевые компоненты:

$$
\hat{p}_{2 x}\left(\vec{\kappa}_{1}\right)=\frac{D}{2}, \quad \hat{p}_{2 z}\left(\vec{\kappa}_{1}\right)=\frac{D}{2}, \quad \hat{p}_{1 z}\left(\vec{\kappa}_{2}\right)=-\frac{D}{2}, \quad \hat{p}_{1 y}\left(\vec{\kappa}_{2}\right)=-\frac{D}{2} .
$$

Учитывая формулы (71)-(72) находим поле скорости нулевого приближения

$$
\begin{aligned}
& u_{0}=C_{1} e^{i \phi_{1}}+A_{2} e^{i \phi_{2}}+\text { c.c. }=u_{01}+u_{02}+u_{03}+u_{04}, \\
& v_{0}=A_{1} e^{i \phi_{1}}-C_{2} e^{i \phi_{2}}+\text { c.c. }=v_{01}+v_{02}+v_{03}+v_{04}, \\
& w_{0}=-C_{1} e^{i \phi_{1}}+C_{2} e^{i \phi_{2}}+\text { c.c. }=w_{01}+w_{02}+w_{03}+w_{04} .
\end{aligned}
$$

Здесь $w_{02}=\left(w_{01}\right)^{*}, w_{04}=\left(w_{03}\right)^{*}, v_{02}=\left(v_{01}\right)^{*}, v_{04}=\left(v_{03}\right)^{*}, u_{02}=\left(u_{01}\right)^{*}, u_{04}=\left(u_{03}\right)^{*}$ и введены новые обозначения:

$$
A_{1,2}=\frac{f_{0}}{2} \frac{a_{1,2}}{a_{1,2}^{2}+\frac{D^{2}}{2}}, \quad C_{1,2}=\frac{f_{0}}{4} \frac{D}{a_{1,2}^{2}+\frac{D^{2}}{2}}, \quad a_{1,2}=\hat{D}_{W_{1,2}}^{*}+\tilde{Q} \frac{H_{1,2}^{2}}{\hat{D}_{H_{1,2}}^{*}} .
$$

В предельном случае $\sigma=0$ неэлектропроводной жидкости формулы (73)-(75) совпадают с результатами работы [41]. Перейдем теперь к вычислениям мелкомасштабных осцилляций поля $\vec{B}_{0}$, используя при этом выражения (59) и (73)-(75):

$$
\begin{aligned}
& B_{0}^{x}=\tilde{u}_{0}=\widetilde{C}_{1} e^{i \varphi_{1}}+\widetilde{A}_{2} e^{i \varphi_{2}}+\text { c.c. }=\tilde{u}_{01}+\tilde{u}_{02}+\tilde{u}_{03}+\tilde{u}_{04}, \\
& B_{0}^{y}=\tilde{v}_{0}=\widetilde{A}_{1} e^{i \varphi_{1}}-\widetilde{C}_{2} e^{i \varphi_{2}}+\text { c.c. }=\tilde{v}_{01}+\tilde{v}_{02}+\tilde{v}_{03}+\tilde{v}_{04}, \\
& B_{0}^{z}=\widetilde{w}_{0}=-\widetilde{C}_{1} e^{i \varphi_{1}}+\widetilde{C}_{2} e^{i \varphi_{2}}+\text { c.c. }=\tilde{w}_{01}+\tilde{w}_{02}+\widetilde{w}_{03}+\widetilde{w}_{04} .
\end{aligned}
$$

Между коэффициентами $\widetilde{A}_{1,2}, \widetilde{C}_{1,2}$ и $A_{1,2}, C_{1,2}$ имеется следующая связь:

$$
\tilde{A}_{1,2}=A_{1,2} \frac{i H_{1,2}}{\hat{D}_{H_{1,2}}^{*}}, \quad \tilde{C}_{1,2}=C_{1,2} \frac{i H_{1,2}}{\hat{D}_{H_{1,2}}^{*}}
$$

В заключении данного раздела отметим, что найденные здесь выражения для мелкомасштабных осцилляций поля скорости $\vec{v}_{0}$ и магнитного поля $\vec{B}_{0}$ будут использоваться для вычислений корреляционных функций. 
ПРИЛОЖЕНИЕ III.

ВЫЧИСЛЕНИЕ НАПРЯЖЕНИЙ РЕЙНОЛЬДСА, МАКСВЕЛЛА И ТУРБУЛЕНТНОЙ Э.Д.С.

Для замыкания системы уравнений (17)-(20), описывающих зволюцию крупномасштабных полей, необходимо вычислить корреляторы следующего вида:

$$
\begin{aligned}
& T^{31}=\overline{w_{0} u_{0}}=\overline{w_{01}\left(u_{01}\right)^{*}}+\overline{\left(w_{01}\right)^{*} u_{01}}+\overline{w_{03}\left(u_{03}\right)^{*}}+\overline{\left(w_{03}\right)^{*} u_{03}} \\
& T^{32}=\overline{w_{0} v_{0}}=\overline{w_{01}\left(v_{01}\right)^{*}}+\overline{\left(w_{01}\right)^{*} v_{01}}+\overline{w_{03}\left(v_{03}\right)^{*}}+\overline{\left(w_{03}\right)^{*} v_{03}} \\
& S^{31}=\overline{\widetilde{w}_{0} \tilde{u}_{0}}=\overline{\tilde{w}_{01}\left(\tilde{u}_{01}\right)^{*}}+\overline{\left(\tilde{w}_{01}\right)^{*} \tilde{u}_{01}}+\overline{\tilde{w}_{03}\left(\tilde{u}_{03}\right)^{*}}+\overline{\left(\tilde{w}_{03}\right)^{*} \tilde{u}_{03}} \\
& S^{32}=\overline{\tilde{w}_{0} \tilde{v}_{0}}=\overline{\tilde{w}_{01}\left(\tilde{v}_{01}\right)^{*}}+\overline{\left(\tilde{w}_{01}\right)^{*} \tilde{v}_{01}}+\overline{\tilde{w}_{03}\left(\tilde{v}_{03}\right)^{*}}+\overline{\left(\tilde{w}_{03}\right)^{*} \tilde{v}_{03}} \\
& G^{13}=\overline{u_{0} \tilde{w}_{0}}=\overline{u_{01}\left(\tilde{w}_{01}\right)^{*}}+\overline{\left(u_{01}\right)^{*} \tilde{w}_{01}}+\overline{u_{03}\left(\tilde{w}_{03}\right)^{*}}+\overline{\left(u_{03}\right)^{*} \tilde{w}_{03}} \\
& G^{31}=\overline{w_{0} \tilde{u}_{0}}=\overline{w_{01}\left(\tilde{u}_{01}\right)^{*}}+\overline{\left(w_{01}\right)^{*} \tilde{u}_{01}}+\overline{w_{03}\left(\tilde{u}_{03}\right)^{*}}+\overline{\left(w_{03}\right)^{*} \tilde{u}_{03}} \\
& G^{32}=\overline{\tilde{v}_{0} \tilde{w}_{0}}=\overline{\tilde{v}_{01}\left(\tilde{w}_{01}\right)^{*}}+\overline{\left(v_{01}\right)^{*} \tilde{w}_{01}}+\overline{v_{01}\left(\tilde{w}_{01}\right)^{*}}+\overline{\left(v_{03}\right)^{*} \tilde{w}_{03}}
\end{aligned}
$$

В формулах (81)-(88), для удобства приняты новые обозначения мелкомасштабных полей, введенные в Приложении II. Начнем с вычислений напряжений Рейнольдса, определяемых формулами (81), (82). Прежде всего нам потребуются выражения для мелкомасштабных полей скорости (73)-(75) (см. Приложение II), после подстановки которых в формулы (81), (82) получим:

$$
\begin{aligned}
& T^{31}=-2\left|C_{1}\right|^{2}+C_{2} A_{2}^{*}+C_{2}^{*} A_{2}, \\
& T^{32}=-2\left|C_{2}\right|^{2}-\left(C_{1} A_{1}^{*}+C_{1}^{*} A_{1}\right) .
\end{aligned}
$$

Теперь приступим к вычислению корреляторов магнитного поля или напряжений Максвелла $S^{31}$ и $S^{32}$, используя при этом формулы (77)-(79) из Приложения II. Тогда в результате подстановки (77)-(79) в (83), (84) находим:

$$
\begin{aligned}
& S^{31}=-2\left|C_{1}\right|^{2} \frac{H_{1}^{2}}{\left|\hat{D}_{H_{1}}\right|^{2}}+\left(C_{2} A_{2}^{*}+C_{2}^{*} A_{2}\right) \frac{H_{2}^{2}}{\left|\hat{D}_{H_{2}}\right|^{2}}, \\
& S^{32}=-2\left|C_{2}\right|^{2} \frac{H_{2}^{2}}{\left|\hat{D}_{H_{2}}\right|^{2}}-\left(C_{1} A_{1}^{*}+C_{1}^{*} A_{1}\right) \frac{H_{1}^{2}}{\left|\hat{D}_{H_{1}}\right|^{2}} .
\end{aligned}
$$

Поскольку в правых частях уравнений (17), (18) содержится разность $T^{31}-\tilde{Q} S^{31}$ и $T^{32}-\tilde{Q} S^{32}$, то ее несложно найти воспользовавшись выражениями (89)-(92):

$$
T^{31}-\tilde{Q} S^{31}=-2\left|C_{1}\right|^{2}\left(1-\frac{Q H_{1}^{2}}{P m\left|\hat{D}_{H_{1}}\right|^{2}}\right)+\left(C_{2} A_{2}^{*}+C_{2}^{*} A_{2}\right)\left(1-\frac{Q H_{2}^{2}}{P m\left|\hat{D}_{H_{2}}\right|^{2}}\right)
$$




$$
T^{32}-\tilde{Q} S^{32}=-2\left|C_{2}\right|^{2}\left(1-\frac{Q H_{2}^{2}}{P m\left|\hat{D}_{H_{2}}\right|^{2}}\right)-\left(C_{1} A_{1}^{*}+C_{1}^{*} A_{1}\right)\left(1-\frac{Q H_{1}^{2}}{P m\left|\hat{D}_{H_{1}}\right|^{2}}\right) .
$$

Используя формулы (71) и (76) выпишем ряд полезных соотношений:

$$
\left|C_{1,2}\right|^{2}=C_{1,2} C_{1,2}^{*}=\frac{f_{0}^{2}}{16} \frac{D^{2}}{\left[16\left(1-W_{1,2}\right)^{2}+\left[\frac{D^{2}}{2}+4-\left(1-W_{1,2}\right)^{2}\right]^{2}+h_{1,2}\right]},
$$

где введены обозначения:

$$
\begin{gathered}
h_{1,2}=2 f_{1,2}\left(4+\left(1-W_{1,2}\right)^{2}\right)^{2}+f_{1,2}^{2}\left(4+\left(1-W_{1,2}\right)^{2}\right)^{2}+D^{2} g_{1,2}, \\
g_{1,2}=\frac{Q H_{1,2}^{2}}{\left(4+P m^{2}\left(1-W_{1,2}\right)^{2}\right)}\left[2\left(4+P m\left(1-W_{1,2}\right)^{2}\right)+\frac{Q H_{1,2}^{2}\left(4-P m^{2}\left(1-W_{1,2}\right)^{2}\right)}{4+P m^{2}\left(1-W_{1,2}\right)^{2}}\right], \\
f_{1,2}=\frac{Q H_{1,2}^{2}}{\left(4+\left(1-W_{1,2}\right)^{2}\right)\left(4+P m^{2}\left(1-W_{1,2}\right)^{2}\right)}\left[2\left(4+P m\left(1-W_{1,2}\right)^{2}\right)+Q H_{1,2}^{2}\right], \\
C_{1,2} A_{1,2}^{*}+C_{1,2}^{*} A_{1,2}=\frac{f_{0}^{2}}{2} \frac{D q_{1,2}}{\left[16\left(1-W_{1,2}\right)^{2}+\left[\frac{D^{2}}{2}+4-\left(1-W_{1,2}\right)^{2}\right]^{2}+h_{1,2}\right]}, \\
q_{1,2}=1+\frac{Q H_{1,2}^{2}}{4+P m^{2}\left(1-W_{1,2}\right)^{2}} .
\end{gathered}
$$

С учетом соотношений (95), (96) формулы (93), (94) запишем в явном виде:

$$
\begin{aligned}
& T^{31}-\tilde{Q} S^{31}=\frac{f_{0}^{2}}{2} \frac{D q_{2}\left(1+P m\left(1-q_{2}\right)\right)}{\left[16\left(1-W_{2}\right)^{2}+\left[\frac{D^{2}}{2}+4-\left(1-W_{2}\right)^{2}\right]^{2}+h_{2}\right]}- \\
& -\frac{f_{0}^{2}}{8} \frac{D^{2}\left(1+P m\left(1-q_{1}\right)\right)}{\left[16\left(1-W_{1}\right)^{2}+\left[\frac{D^{2}}{2}+4-\left(1-W_{1}\right)^{2}\right]^{2}+h_{1}\right]} \\
& T^{32}-\tilde{Q} S^{32}=-\frac{f_{0}^{2}}{8} \frac{D^{2}\left(1+P m\left(1-q_{2}\right)\right)}{\left[16\left(1-W_{2}\right)^{2}+\left[\frac{D^{2}}{2}+4-\left(1-W_{2}\right)^{2}\right]^{2}+h_{2}\right]} \\
& -\frac{f_{0}^{2}}{2} \frac{D q_{1}\left(1+P m\left(1-q_{1}\right)\right)}{\left[16\left(1-W_{1}\right)^{2}+\left[\frac{D^{2}}{2}+4-\left(1-W_{1}\right)^{2}\right]^{2}+h_{1}\right]}
\end{aligned} .
$$


Отсюда находим, что коэффициенты нелинейного гидродинамического $\alpha$-эффекта имеют следующий вид:

$$
\begin{aligned}
& \alpha_{(1)}=\frac{f_{0}^{2}}{2} \frac{D q_{2}\left(1+P m\left(1-q_{2}\right)\right)\left(1-W_{2}\right)^{-1}}{\left[16\left(1-W_{2}\right)^{2}+\left[\frac{D^{2}}{2}+4-\left(1-W_{2}\right)^{2}\right]^{2}+h_{2}\right]}-\frac{f_{0}^{2}}{8} \frac{D^{2}\left(1+P m\left(1-q_{1}\right)\right)\left(1-W_{2}\right)^{-1}}{\left[16\left(1-W_{1}\right)^{2}+\left[\frac{D^{2}}{2}+4-\left(1-W_{1}\right)^{2}\right]^{2}+h_{1}\right]}, \\
& \alpha_{(2)}=\frac{f_{0}^{2}}{2} \frac{D q_{1}\left(1+P m\left(1-q_{1}\right)\right)\left(1-W_{1}\right)^{-1}}{\left[16\left(1-W_{1}\right)^{2}+\left[\frac{D^{2}}{2}+4-\left(1-W_{1}\right)^{2}\right]^{2}+h_{1}\right]}+\frac{f_{0}^{2}}{8} \frac{D^{2}\left(1+P m\left(1-q_{2}\right)\right)\left(1-W_{1}\right)^{-1}}{\left[16\left(1-W_{2}\right)^{2}+\left[\frac{D^{2}}{2}+4-\left(1-W_{2}\right)^{2}\right]^{2}+h_{2}\right]} .
\end{aligned}
$$

В предельном случае $\sigma=0$ неэлектропроводящей жидкости формулы (97)-(98) переходят к уже известным результатам из работы [41] для напряжений Рейнольдса. Переходим к вычислениям последней группы корреляторов, определяемых формулами (85)-(88). Для этого нам потребуются выражения как для мелкомасштабного поля скорости (73)-(75), так и для магнитного поля (77)-(79). В результате проведения несложных математических операций находим:

$$
\begin{gathered}
G^{13}=\left|C_{1}\right|^{2} i H_{1} \frac{\left(\widehat{D}_{H_{1}}^{*}-\widehat{D}_{H_{1}}\right)}{\left|\widehat{D}_{H_{1}}\right|^{2}}+i H_{2}\left(\frac{A_{2}^{*} C_{2}}{\widehat{D}_{H_{2}}^{*}}-\frac{A_{2} C_{2}^{*}}{\widehat{D}_{H_{2}}}\right), \\
G^{31}=\left|C_{1}\right|^{2} i H_{1} \frac{\left(\widehat{D}_{H_{1}}^{*}-\widehat{D}_{H_{1}}\right)}{\left|\widehat{D}_{H_{1}}\right|^{2}}+i H_{2}\left(\frac{A_{2} C_{2}^{*}}{\widehat{D}_{H_{2}}^{*}}-\frac{A_{2}^{*} C_{2}}{\widehat{D}_{H_{2}}}\right) \\
G^{23}=\left|C_{2}\right|^{2} i H_{2} \frac{\left(\widehat{D}_{H_{2}}^{*}-\widehat{D}_{H_{2}}\right)}{\left|\widehat{D}_{H_{2}}\right|^{2}}+i H_{1}\left(\frac{A_{1} C_{1}^{*}}{\widehat{D}_{H_{1}}}-\frac{A_{1}^{*} C_{1}}{\widehat{D}_{H_{1}}^{*}}\right) \\
G^{32}=\left|C_{2}\right|^{2} i H_{2} \frac{\left(\widehat{D}_{H_{2}}^{*}-\widehat{D}_{H_{2}}\right)}{\left|\widehat{D}_{H_{2}}\right|^{2}}+i H_{1}\left(\frac{A_{1}^{*} C_{1}}{\widehat{D}_{H_{1}}}-\frac{A_{1} C_{1}^{*}}{\widehat{D}_{H_{1}}^{*}}\right)
\end{gathered}
$$

Для замыкания уравнений крупномасштабного магнитного поля (19), (20) нам нужно вычислить разности $G^{13}-G^{31}, G^{23}-G^{32}$, которые соответствуют компонентам турбулентной э.д.с. $\varepsilon_{2}=\varepsilon_{y}$ и $\varepsilon_{1}=\varepsilon_{x}$. С учетом формул (99)-(102) получим:

$$
\begin{aligned}
& \mathcal{E}_{2}=G^{13}-G^{31}=i\left(\frac{\hat{D}_{H_{2}}^{*}+\hat{D}_{H_{2}}}{\left|\hat{D}_{H_{2}}\right|^{2}}\right)\left(A_{2}^{*} C_{2}-A_{2} C_{2}^{*}\right) H_{2}, \\
& \mathcal{E}_{1}=G^{23}-G^{32}=-i\left(\frac{\hat{D}_{H_{1}}^{*}+\hat{D}_{H_{1}}}{\left|\hat{D}_{H_{1}}\right|^{2}}\right)\left(A_{1}^{*} C_{1}-A_{1} C_{1}^{*}\right) H_{1} .
\end{aligned}
$$


Зная определение обозначений $A_{1,2}, C_{1,2}, \hat{D}_{H_{1,2}}$ из формул (71), (76) (см. Приложение II), находим компоненты турбулентной э.д.с. $\mathcal{E}_{2}$ и $\mathcal{E}_{1}$ в явном виде:

$$
\begin{aligned}
& \mathcal{E}_{2}=-f_{0}^{2} \frac{D\left(1-W_{2}\right) P m\left(1+P m\left(1-q_{2}\right)\right) H_{2}}{\left(4+P m^{2}\left(1-W_{2}\right)^{2}\right)\left[16\left(1-W_{2}\right)^{2}+\left[\frac{D^{2}}{2}+4-\left(1-W_{2}\right)^{2}\right]^{2}+h_{2}\right]}=-\alpha_{H}^{(2)} H_{2}, \\
& \mathcal{E}_{1}=f_{0}^{2} \frac{D\left(1-W_{1}\right) P m\left(1+P m\left(1-q_{1}\right)\right) H_{1}}{\left(4+P m^{2}\left(1-W_{1}\right)^{2}\right)\left[16\left(1-W_{1}\right)^{2}+\left[\frac{D^{2}}{2}+4-\left(1-W_{1}\right)^{2}\right]^{2}+h_{1}\right]}=\alpha_{H}^{(1)} H_{1} .
\end{aligned}
$$

Здесь $\alpha_{H}^{(1)}, \alpha_{H}^{(2)}$ соответствуют коэффициентам нелинейного магнитогидродинамического эффекта.

\section{REFERENCES}

1. Moffat G. Vozbuzdenie magnitnogo polja v provodjashej srede [Magnetic Field Generation in Electrically Conducting Fluids]. - M.: Mir, 1980. - 343p. (in Russian)

2. Zeldovitsh Ja.B., Ruzmajkin A.A., Sokolov D.D. Magnitnye polja v astrofizike [Magnetic Fields in Astrophysics]. Izhevsk: Inst. Kopm.issl. RHD, 2006. - 384 p. (in Russian)

3. Vajnshtejn S.I. , Zeldovitsh Ja.B., Ruzmajkin A.A. Turbulentnoe dinamo v astrophisike [Turbulent Dynamo in Astrophysics]. - M.:Nauka, 1980. - 237p. (in Russian)

4. Vajnshtejn S.I. Magnitnye polja $v$ kosmose [ Magnetic fields in Space]. - M.:Nauka, 1983. - 237p. (in Russian)

5. Parker Ju. Besedy ob elektritseskih i magnitnyh poljah v kosmose [Conversations on Electric and Magnetic Fields in Space]. - Izhevsk: Inst. Kopm.issl. RHD, 2010.- 208 p. (in Russian)

6. Krauze F., Pedler K.H. Magnitnaja gidrodinamika srednih polej i teorija dinamo [Mean-Field Magnetohydrodynamics and Dynamo Theory ]. - M.: Mir, 1984. - 314p. (in Russian)

7. Ruzmajkin A.A., Sokolov D.D., Shukurov A.M. Magnitnye polja galaktik [Magnetic fields of Galaxies]. - M.: Nauka, 1988. - 279p. (in Russian)

8. Sokolov D.D., Stepanov R.A., Frik P.G. Dinamo na puti ot astrofizitseskih modelej k labolatornomu eksperimentu[Dynamo: from an Astrophysical Model to Laboratory Experiments] // Usp. Fiz.Nauk. - 2014. - Vol. 184. - P.318-335. (in Russian)

9. Arnol'd V.I., Zel'dvich Ja.B., Ruzmajkin A.A., Sokolov D.D. Magnitnoe pole v dvizhushejsija provodashej zhidkosti [Magnetic Field in a Moving Conducting Fluid] // Uspehi matem. Nauk. - 1981. - No5. - P.220-221. (in Russian)

10. Arnol'd V.I. Evolutsija magnitnogo polija pod deijstviem perenosa I diffuzii [Evolution of Magnetic Field under the Action of Transfer and Diffusion ] // Uspehi matem. Nauk. - 1983. - No. 2. - P.225-227. (in Russian)

11. Grinspen H. Teorija vrashajushihsija zhidkostej [The Theory of Rotating Fluids]. Per. S angl. - Leningrad: Gidrometeoizdat, 1975. - 304p. (in Russian)

12. Reshetnyak M.Yu. Thermal Convection and the Dynamo during Rapid Rotation // Izvestija. Physics of the solid Earth.-2007. - Vol.43. - No.8. - P.642-652.

13. Pedloski Dj. Geofizicheskaija gidrodinamika v 2-h t. Per. s angl. [Geophysical Fluid Dynamics] - M.: Mir, 1984. (in Russian)

14. Petviashvili V.I., Pohotelov O.A. Uedinenye vihri v plasme i atmosfere [Solitary waves in Plasma and Atmosphere].- M.:Energoatomisdat, 1989. - 200p. (in Russian)

15. Aburdjanija G.D. Samoorganisatsia nelinejnyh vihrevyh struktur i vihrevoj turbulentnosti v dispergirujuscih sredah [Self-organization of Nonlinear Vortex Structures and Vortex Turbulence in Dispersive Media]. -M.:KomKniga, 2006. - 328p. (in Russian)

16. Kolesnichenko A.V., Marov M.Ja. Turbulentnost' i samoorganizatsija. Problemy modelirovanija kosmicheskikh i prirodnykh sred [Turbulence and self-organization. Problems of modeling space and natural environments]. - M.: BINOM, 2009. - 632p. (in Russian)

17. Onishenko O.G., Pohotelov O.A., Astafjeva N.M. Generatsyja krupnomasshtabnyh vihrej i sonalnykh vetrov v atmosferakh planet [Generation of Large-Scale Vortices and Zonal Winds in Planetary Atmospheres] // Usp. Fis. Nauk. - 2008. - Vol.178. - No. 6 - P. 605 - 618. (in Russian)

18. Nezlin M.V., Sneshkin E.N. Vikhri Rossby i spiral'nye struktury [Rossby Vortices and Spiral Structure ]. - M.: Nauka, 1990. - 240p. (in Russian)

19. Rudiger G. On the $\alpha$ - Effect for Slow and Fast Rotation // Astron. Nachr. - 1978. - Vol.299. - No.4. - P.217-222. 
20. Steenbek M., Krause F. Vozniknovenie magnitnyh polej zvezd i planet v resul'tate turbulentnogo dvizhenija ih veshestv [The Origin of the Magnetic Fields of Stars and Planets as a Result of Turbulent Movement of their Substances] // Magnitnaja gidrodinamika. - 1967. - No.3. - P.19-44. (in Russian)

21. Moiseev S.S., Sagdeev R.Z., Tur A.V., Homenko G.A., Yanovsky V.V. Teorija vozniknovenija krupnomasshtabnykh struktur $\mathrm{v}$ gidrodiamitsheskoj turbulentnosti [The Theory of the Origin of Large-Scale Structures in Hydrodynamic Turbulence] // ZhETF. - 1983. - Vol.85. - Iss.6(12). - P.1979-1987. (in Russian)

22. Gilbert A. D., Frisch U., and Pouquet A. Geophys. //And Astrophys. Fluid Dyn.-1988.-Vol. 42.-P. 151-167.

23. Krause F., Rudiger G. On the Reynolds stresses in mean-field hydrodynamics. I. Incompressible homogeneous isotropic turbulence // Astron. Nachr. - 1974. Vol. 295. - P.93-99.

24. Chkhetiani O. G., Moiseev S. S., Petrosyan A. S., Sagdeev R. Z. The large scale stability and self-organization in homogeneous turbulent shear fow // Physica Scripta. - 1994. - Vol.49. - P.214-220.

25. Moiseev S.S., Rutkevith P.B., Tur A.V., Homenko G.A., Yanovsky V.V. Vortex dynamos in a helical turbulent convection // Sov.Phys.JETP.- 1988. - Vol. 67. - P. 294 -299.

26. Lypyan E.A., Mazurov A.A., Rutkevitch P.B., Tur A.V. Generation of large-scale vortices through the action of spiral turbulence of a convective nature // Sov.Phys.JETP. - 1992. -Vol. 75.- P.838-841.

27. Moiseev S.S., Oganjan O.R., Rutkevith P.B., Tur A.V., Homenko G.A., Yanovsky V.V. Vikhrevoe dinamo v spiral'noj turbulentnostju [Vortex Dynamo in Helical Turbulence] - Kiev: Naukova dumka. Sb.: Integriruemost i kinetitcheskie uravnenija dlja solitonov, 1990. - P.280-382. (in Russian)

28. Levina G.V., Moiseev S.S., Rutkevitch P.B. Hydrodynamic alpha-effect in a convective system // Advance in Fluid Mechanics.-2000.-Vol. 25.-P. 111.

29. Zimin V.D., Levina G.V., Moiseev S.S., Tur A.V. Vozniknovenie krupnomasshtabnych struktur pri turbulentnoy konvektsij v podogrevaevom snizu vrashajushemssja sloe [The Emergence of Large-Scale Structures under Turbulent Convection in a heated from below Rotating Layer] // Dokl. AN SSSR. - 1989. - Vol. 309. - P. 88-92. (in Russian)

30. Tur A.V., Yanovsky V.V. Large-scale instability in hydrodynamics with stable temperature stratification driven by small-scale helical force//arXiv:1204.5024 v.1 (physics.flu-dyn.)(2012).

31. Tur A.V., Yanovsky V.V. Non Linear Vortex Structure in Stratified Driven by Small- scale Helical Forse // Open Journal of Fluid Dynamics. - 2013. - Vol. 3 - P. 64-74.

32. Kopp M.I., Tur A. V., Yanovsky V. V. Krupnomasshtabnaja konvektivnaja neustojtchivost' v elektroprovodjashej srede s melkomasshtabnoj spiral'noj turbulentnostyju [Large-Scale Convective Instability in an Electroconducting Medium with Small Scale Helicity ] // ZhETF. - 2015. - Vol. 147. - Iss. 4. - P.846-866. (in Russian)

33. Rutkevich P. B. Equation for vortex Instability Caused by Convective Turbulence and Coriolis Force.// Sov.Phys. JETF. - 1993.- Vol. 77.- P.933.

34. Smith L. M. and Waleffe F. Transfer of Energy to Two-Dimensional Large Scales in Forced, Rotating ThreeDimensional Turbulence// Physics of Fluids.- 1999.- Vol. 11.- No. 6.- P.1608.

35. Smith L. M. and Waleffe F. Generation of Slow Large Scales in Forced Rotating Stratified Turbulence // Journal of Fluid Mechanics. - 2002. - Vol.451. - P.145 -168.

36. Berezin Y. A. and Zhukov V. P. An Influence of Rotation on Convective Stability of Large Scale Distorbances in Turbulent Fluid // Izv. AN SSSR. Mech. Zhidk. Gaza. - 1989. - No. 4. - P.3.

37. Kitchatinov L.L., Rudiger G., and G.Khomenko. Large -scale vortices in rotating stratified disks // Astron. Astrophys. - 1994. -Vol.287. - P.320-334.

38. Frishe U., She Z.S., Sulem P.L. Large Scale Flow Driven by the Anisotropic Kinetic Alpha Effect // Physica 28D. 1987. - P. 283 - 291.

39. Druzhinin O.A., Homenko G.A. Nelinejnaja teorija gidrodinamitsheskogo alfa-effekta v szhimaemoj srede i obratny kaskad energii [Nonlinear Theory of Hydrodynamic Alpha-Effect in the Compressible Fluid and the inverse Energy Cascade]. - Kiev: Naukova dumka. Tr. Mezhdunarodnoj konf.: Nelinejnye i turbulentnye protsessy v fizike, 1989. - Vol.2. - P.83 -86. (in Russian)

40. Rutkevitch P.B., Sagdeev R.Z., Tur A.V., Yanovsky V.V. Nonlinear dynamic theory of the $\alpha$-effect in compressible fluid. - Kiev: Naukova dumka. Proceeding of the IV Intern.Workshop on Nonlinear and Turb. Pros.in Physics, 1989.

41. Kopp M., Tur A., Yanovsky V. The Large Scale Instability in Rotating Fluid with Small Scale Force // Open Journal of Fluid Dynamics.- 2015.- Vol. 5.- P. 128-138.

42. Dolginov A.Z., Urpin V.A. Termomagnitnaja neustojtchivost' neodnorodnoj plazmy [Thermomagnetic Instability of an Inhomogeneous Plasma] // ZhETF. - 1978. - Vol.77. - P.1921-1932. (in Russian) 